\title{
Thermal Pressure in the Cold Neutral Medium of Nearby Galaxies
}

\author{
R. Herrera-Camus ${ }^{1,2}$, A. Bolatto ${ }^{2}$, M. Wolfire ${ }^{2}$, E. Ostriker ${ }^{3}$, B. Draine ${ }^{3}$, A. Leroy ${ }^{4}$, K. Sandstrom ${ }^{5}$, L. Hunt ${ }^{5,14}$, R. Kennicutt ${ }^{6}$, \\ D. Calzetti ${ }^{7}$, J. D. Smith $^{8}$, K. Croxall ${ }^{4}$, M. Galametz ${ }^{9}$, I. de Looze ${ }^{10}$, D. Dale ${ }^{11}$, A. Crocker ${ }^{12}$, and B. Groves ${ }^{13}$ \\ ${ }^{1}$ Max-Planck-Institut für Extraterrestrische Physik (MPE), Giessenbachstr., D-85748 Garching, Germany \\ ${ }^{2}$ Department of Astronomy, University of Maryland, College Park, MD 20742, USA \\ ${ }^{3}$ Department of Astrophysical Sciences, Princeton University, Princeton, NJ 08544, USA \\ ${ }^{4}$ Department of Astronomy, The Ohio State University, 4051 McPherson Laboratory, 140 West 18th Avenue, Columbus, OH 43210, USA \\ ${ }^{5}$ Center for Astrophysics and Space Sciences, Department of Physics, University of California, San Diego, 9500 Gilman Drive, La Jolla, CA 92093, USA \\ ${ }^{6}$ Institute of Astronomy, University of Cambridge, Madingley Road, Cambridge CB3 0HA, UK \\ ${ }^{7}$ Department of Astronomy, University of Massachusetts, Amherst, MA 01003, USA \\ ${ }^{8}$ Department of Physics and Astronomy, University of Toledo, 2801 West Bancroft Street, Toledo, OH 43606, USA \\ ${ }^{10}$ European Southern Observatory, Karl Schwarzschild Strasse 2, D-85748 Garching, Germany \\ ${ }^{11}$ Department of Physics and Astronomy, University of Wyoming, Laramie, WY 82071, USA \\ ${ }^{12}$ Department of Physics, Reed College, Portland, OR 97202, USA \\ ${ }^{13}$ Research School of Astronomy and Astrophysics, Australian National University, Canberra, ACT 2611, Australia \\ ${ }^{14}$ INAF-Osservatorio Astrofisico di Arcetri, Largo E. Fermi 5, I-50125 Firenze, Italy \\ Received 2016 November 7; revised 2016 December 8; accepted 2016 December 20; published 2017 January 31
}

\begin{abstract}
Dynamic and thermal processes regulate the structure of the multiphase interstellar medium (ISM), and ultimately establish how galaxies evolve through star formation. Thus, to constrain ISM models and better understand the interplay of these processes, it is of great interest to measure the thermal pressure $\left(P_{\mathrm{th}}\right)$ of the diffuse, neutral gas. By combining [C II] $158 \mu \mathrm{m}, \mathrm{HI}$, and CO data from 31 galaxies selected from the Herschel KINGFISH sample, we have measured thermal pressures in 534 predominantly atomic regions with typical sizes of $\sim 1$ kiloparsec. We find a distribution of thermal pressures in the $P_{\mathrm{th}} / k \sim 10^{3}-10^{5} \mathrm{~K} \mathrm{~cm}^{-3}$ range. For a sub-sample of regions with conditions similar to those of the diffuse, neutral gas in the Galactic plane, we find thermal pressures that follow a log-normal distribution with a median value of $P_{\mathrm{th}} / k \approx 3600 \mathrm{~K} \mathrm{~cm}^{-3}$. These results are consistent with thermal pressure measurements using other observational methods. We find that $P_{\text {th }}$ increases with radiation field strength and star formation activity, as expected from the close link between the heating of the gas and the star formation rate. Our thermal pressure measurements fall in the regime where a two-phase ISM with cold and warm neutral media could exist in pressure equilibrium. Finally, we find that the midplane thermal pressure of the diffuse gas is about $\sim 30 \%$ of the vertical weight of the overlying ISM, consistent with results from hydrodynamical simulations of self-regulated star formation in galactic disks.
\end{abstract}

Key words: galaxies: ISM - galaxies: star formation - ISM: lines and bands - ISM: structure

\section{Introduction}

In the widely accepted thermal instability model of the multiphase interstellar medium (ISM), most of the neutral atomic gas resides in two distinct phases commonly referred as the cold neutral medium $(\mathrm{CNM} ; T \lesssim 300 \mathrm{~K})$, and the warm neutral medium (WNM; peak temperature around $T \approx 8000 \mathrm{~K}$; Field et al. 1969; Heiles \& Troland 2003; Cox 2005). These two phases coexist in pressure equilibrium in a relatively narrow range of pressure $\left(P_{\min }<P<P_{\max } \approx 3 P_{\min }\right.$; Field et al. 1969) regulated by the thermal balance between heating and radiative cooling (Draine 1978; Wolfire et al. 1995, 2003), and the vertical pressure exerted by the gravitational field (Badhwar \& Stephens 1977; Ostriker et al. 2010; Kim et al. 2011).

The characteristics of the thermal pressure $\left(P_{\mathrm{th}}\right)$ curve depend directly on the gas heating rate, which in turn is a function of the metallicity, the ionization rate of atomic hydrogen, and the FUV radiation field (e.g., Wolfire et al. 1995, 2003). The latter is directly proportional to the star formation activity and illustrates the strong connection between pressure and star formation in the ISM. Blitz \& Rosolowsky (2006) find that the hydrostatic (or dynamical equilibrium) pressure is empirically correlated with the fraction of the neutral gas that is molecular and therefore available to form stars. Ostriker et al. (2010) and
Kim et al. (2011, 2013) consider the connection between thermal pressure and star formation on approximately kiloparsec scales for a model in which the disk evolves into a state of dynamical, thermal, and star formation equilibrium. The analytic model of Ostriker et al. (2010) hypothesizes that if the midplane thermal pressure is higher than $P_{\max }$ or lower than $P_{\min }$, the fraction of the cold gas and the star formation activity evolve in order to bring the midplane pressure close to the average value defined by the two-phase thermal pressure curve. The numerical hydrodynamic ISM/star formation simulations of Kim et al. (2011, 2013) support that these hypotheses are satisfied for a range of galactic environments.

In the Galactic plane, different observational techniques have been employed to characterize the distribution of thermal pressures of the diffuse, neutral gas. Jenkins \& Tripp (2001, 2011) use ultraviolet spectra of local stars to identify absorption features created by neutral carbon (CI). These features can be used to determine the population ratios between the three fine-structure ground electronic state levels of C I, revealing the excitation conditions and thermal pressure of the diffuse gas along the line of sight. Jenkins \& Tripp (2011) find a distribution of thermal pressures that can be well represented by a log-normal distribution that extends from $P_{\mathrm{th}} / k \sim 10^{2}$ to $10^{4.5} \mathrm{~K} \mathrm{~cm}^{-3}$, with a mean value of $P_{\mathrm{th}} / k \approx 3800 \mathrm{~K} \mathrm{~cm}^{-3}$. 


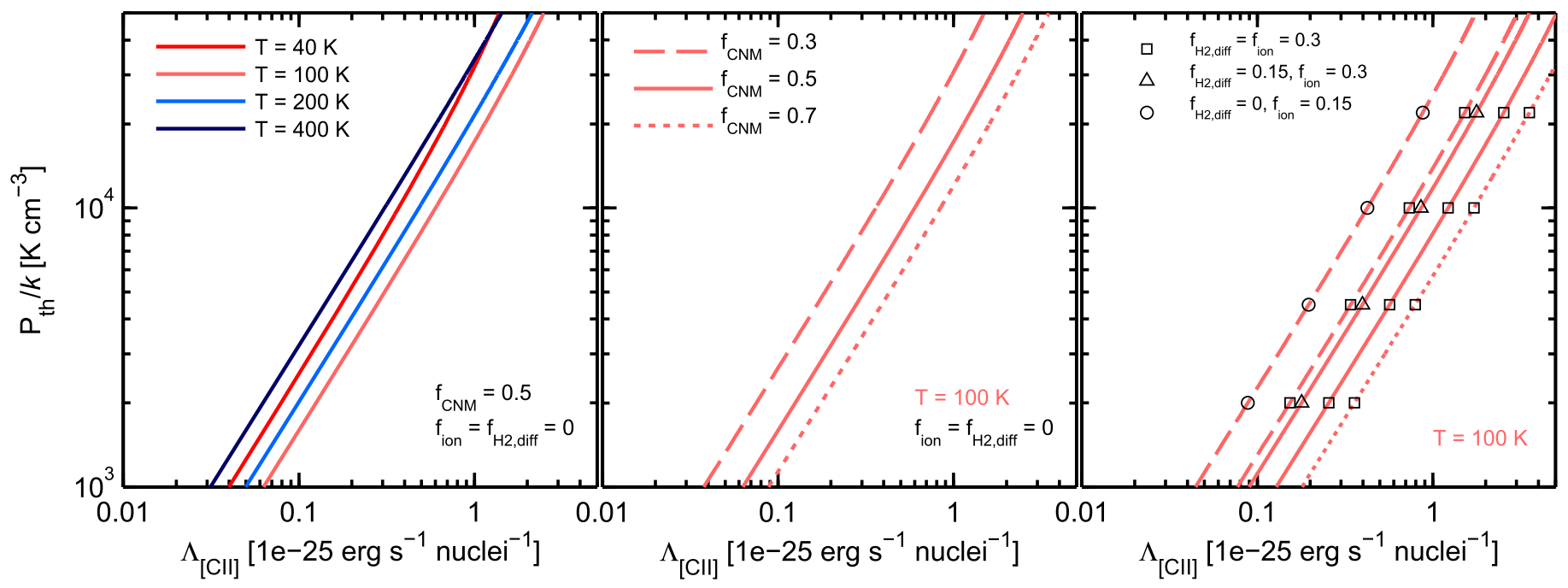

Figure 1. Thermal pressure $P_{\mathrm{th}}$ as a function of the [C II] cooling rate per $\mathrm{H}$ nucleon $\left(\Lambda_{[\mathrm{C} \mathrm{II}}\right.$; Equation (9)) for different assumptions on the temperature of the gas (T), the fraction of the total $N_{\mathrm{H}}$ associated to the CNM phase $\left(f_{\mathrm{CNM}}\right)$, the fraction of the observed [C II] intensity arising from the ionized gas $\left(f_{\text {ion }}\right)$, and the fraction of the diffuse ISM mass contributed by diffuse, "CO-dark" $\mathrm{H}_{2}\left(f_{\mathrm{H}_{2} \text {,diff }}\right)$. For all curves, we have assumed a gas-phase carbon abundance of $(\mathrm{C} / \mathrm{H})$ gas $=1.5 \times 10^{-4}(\mathrm{Gerin}$ et al. 2015) and an electron fraction of $n_{\mathrm{e}} / n_{\mathrm{H}}=4.3 \times 10^{-4}$ (Draine 2011). (Left panel) For a fixed $f_{\mathrm{CNM}}=0.5$ and $f_{\text {ion }}=f_{\mathrm{H}_{2} \text { diff }}=0$, we vary the temperature of the gas for four different values: $T=40 \mathrm{~K}$ (red), $T=100 \mathrm{~K}$ (orange), $T=200 \mathrm{~K}$ (blue), and $T=400 \mathrm{~K}$ (dark blue). (Middle panel) For a fixed temperature of $T=100 \mathrm{~K}$ and $f_{\text {ion }}=f_{\mathrm{H}_{2} \text {,diff }}=0$, we vary the CNM fraction: $f_{\mathrm{CNM}}=0.3$ (dashed), $f_{\mathrm{CNM}}=0.5$ (solid), and $f_{\mathrm{CNM}}=0.7$ (dotted). (Right panel) For a fixed gas temperature of $T=100 \mathrm{~K}$, we show cases where we vary $f_{\mathrm{CNM}}$ (keeping the same line convention that in the middle panel), and $f_{\text {ion }}$ and $f_{\mathrm{H}_{2} \text {,diff }}$ by assuming the following cases: $f_{\text {ion }}=f_{\mathrm{H}_{2} \text {, diff }}=0.3$ (square), $f_{\text {ion }}=0.3, f_{\mathrm{H}_{2} \text {, diff }}=0.15$ (triangle), and $f_{\text {ion }}=0.15, f_{\mathrm{H}_{2} \text {, diff }}=0$ (circle).

Goldsmith (2013), based on ultraviolet measurements of interstellar CO toward nearby stars (Sheffer et al. 2008), calculate thermal pressure values for diffuse interstellar molecular clouds in the $4600-6800 \mathrm{~K} \mathrm{~cm}^{-3}$ range. An additional method to probe the diffuse gas along a particular line of sight is to use [C II] $158 \mu \mathrm{m}$ velocity-resolved observations toward bright infrared continuum sources. This allows, based on the absorption and emission features in the spectra, a measure of the line opacity and the line peak temperature, which in turn can be used to derive the density and thermal pressure of the neutral gas. Using this technique, Gerin et al. (2015) find a median thermal pressure of $P_{\mathrm{th}} / k \approx 5900 \mathrm{~K} \mathrm{~cm}^{-3}$ in 13 lines of sight in the Galactic plane.

On the modeling side, Wolfire et al. (2003) use a comprehensive approach that considers the different sources of heating and cooling of the gas in order to estimate $P_{\min }, P_{\max }$ and the average thermal pressure in the Galactic plane as a function of radius. They conclude that most of the neutral gas in the ISM of the Galaxy out to $\sim 18 \mathrm{kpc}$ have thermal pressures that lie between $P_{\min }$ and $P_{\max }$ (standard $P_{\min }$ and $P_{\max }$ values in the Galactic plane are approximately $2 \times 10^{3}$ and $5 \times 10^{3} \mathrm{~K} \mathrm{~cm}^{-3}$, respectively; Wolfire et al. 2003). Inside the solar circle, they calculate a mean thermal pressure of $P_{\mathrm{th}} /$ $k \approx 3000 \mathrm{~K} \mathrm{~cm}^{-3}$, which is lower than, but consistent with, observational results (Jenkins \& Tripp 2011; Gerin et al. 2015).

In this paper, we use a method developed by Kulkarni \& Heiles (1987) that combines the [C II] $158 \mu \mathrm{m}$ and H I $21 \mathrm{~cm}$ line to measure the $[\mathrm{C} \mathrm{II}]$-cooling rate, the density of the neutral gas and, for a given temperature of the CNM, the thermal pressure in the neutral ISM of nearby galaxies. For CNM temperatures in the range $\sim 40-400 \mathrm{~K}$, the results are quite insensitive to the adopted temperature (e.g., see Figure 1). This paper is organized as follows. In Section 2, we describe the sample of galaxies and the data. In Section 3, we discuss our method to measure thermal pressures using the [C II] and $\mathrm{H} \mathrm{I}$ data. In Section 4, we describe our region selection criteria and the assumptions made in the thermal pressure calculation. In Section 5, we analyze the resulting distribution of thermal pressures. In Section 6, we explore the connection between thermal pressure and star formation activity (and radiation field strength). In Section 7, we measure the total to thermal pressure ratio and we compare it to theoretical predictions. Finally, in Section 8, we present our summary and conclusions.

\section{Main Sample Description}

Our sample consists of 31 galaxies drawn from the KINGFISH sample ("Key Insights on Nearby Galaxies: A Far-Infrared Survey with Herschel"; Kennicutt et al. 2011) that have $\mathrm{CO}$ and $\mathrm{HI}$ observations available from the THINGS ("The H I Nearby Galaxy Survey"; Walter et al. 2008) and HERACLES ("The HERA CO Line Extragalactic Survey"; Leroy et al. 2009) surveys. See Table 1 for a list of the galaxies. With the exception of NGC 3077, which is classified as I0 pec, all of the other galaxies in our sample are spirals. They span a range in total infrared (TIR) luminosity of $L_{\mathrm{TIR}} \sim 10^{8.3}-10^{10.7}$ $L_{\odot}$ (Dale et al. 2012) and in distance of $D \sim 2.8-26.5 \mathrm{Mpc}$. Their metallicities, taken from Moustakas et al. (2010) and measured as the average between the characteristic oxygen abundances from the Pilyugin \& Thuan (2005; PT05) and Kobulnicky \& Kewley (2004; KK04) calibrations (Croxall et al. 2013), are in the $12+\log (\mathrm{O} / \mathrm{H}) \sim 8.1-9.0$ range.

\subsection{KINGFISH [C II]}

In this work, we use [C II] $158 \mu \mathrm{m}$ observations drawn from the Herschel key program KINGFISH (Kennicutt et al. 2011). These were carried out with the Photodetector Array Camera \& Spectrometer (PACS) on board Herschel, and were reduced using the Herschel Interactive Processing Environment (HIPE) version 11.0. For more details on the data reduction process, we refer to Croxall et al. (2013). The angular resolution of the PACS spectrometer at $158 \mu \mathrm{m}$ is $\approx 12^{\prime \prime}$. More than half of the 
Table 1

List of Galaxies Included in this Study

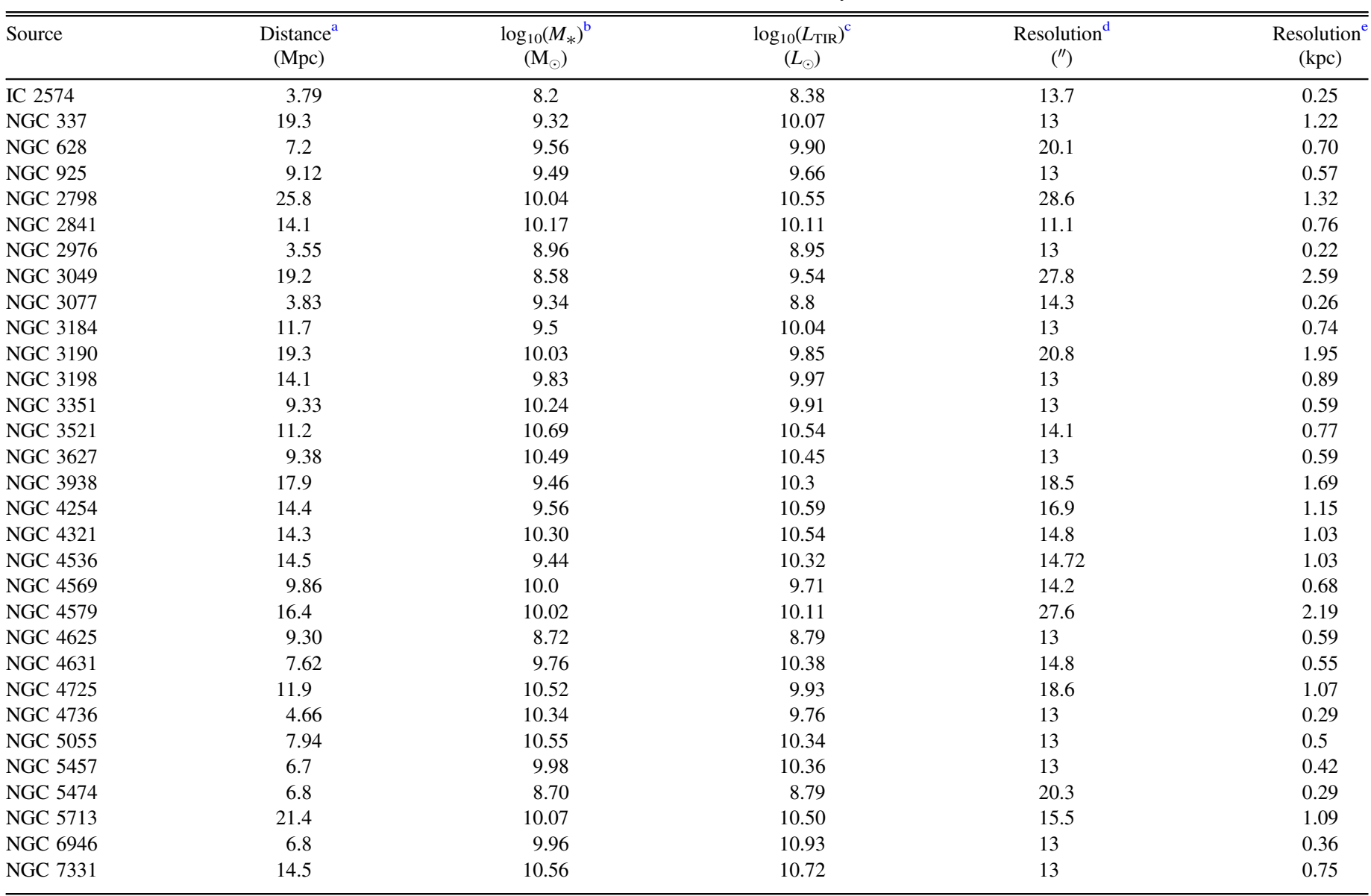

Notes.

a The method of distance determination is given by Kennicutt et al. (2011).

b Total infrared luminosity TIR in the 3-1100 $\mu \mathrm{m}$ range. Fluxes are either from Dale et al. (2007) or Dale et al. (2009).

${ }^{\mathrm{c}}$ Stellar masses obtained from the multi-color method described in Zibetti et al. (2009), and listed in Skibba et al. (2011).

${ }^{\mathrm{d}}$ Common final angular resolution of the convolved [C II], H I, and CO maps.

${ }^{\mathrm{e}}$ Linear resolution corresponding to the final angular resolution at the distance of the target.

[C II] maps consist of a strip that covers the central region of the galaxy and part of the disk. In addition, there are cases where we have coverage of extra-nuclear regions located in the outskirts of the disk (e.g., M101, NGC 6946).

\subsection{THINGS HI}

We retrieve H I maps from the Very Large Array (VLA) THINGS survey (Walter et al. 2008) and a collection of new and archival Karl G. Jansky VLA data (Schruba et al. 2011; Leroy et al. 2013). These have angular resolutions in the $6^{\prime \prime}-$ $25^{\prime \prime}$ range. For more details on the data reduction and map properties, we refer to Walter et al. (2008). We convert the $21 \mathrm{~cm}$ intensities into H I surface densities via

$$
\Sigma_{\mathrm{HI}}\left[M_{\odot} \mathrm{pc}^{-2}\right]=0.02 I_{\mathrm{HI}} \times \cos i\left[\mathrm{~K} \mathrm{~km} \mathrm{~s}^{-1}\right] .
$$

This equation assumes optically thin emission, includes a factor of 1.36 to account for the contribution from helium, and is projected to face-on orientation using inclinations (i) drawn from the compiled list in Kennicutt et al. (2011) and Hunt et al. (2015).

\subsection{HERACLES CO}

We trace the molecular gas using $\mathrm{CO}(J=2 \rightarrow 1)$ observations taken with the Heterodyne Receiver Array (HERA) on the IRAM $30 \mathrm{~m}$ telescope obtained as part of the HERACLES survey (Leroy et al. 2009). The angular resolution of the HERACLES data is about $\sim 13^{\prime \prime}$, similar to the resolution of Herschel using the PACS [C II] observations. We convert the $\mathrm{CO}(2 \rightarrow 1)$ intensities into molecular mass surface densities following

$$
\Sigma_{\mathrm{mol}}\left[M_{\odot} \mathrm{pc}^{-2}\right]=6.25 I_{\mathrm{CO}} \times \cos i\left[\mathrm{~K} \mathrm{~km} \mathrm{~s}^{-1}\right],
$$

where we have assumed a $\mathrm{CO}$ line ratio of $I_{\mathrm{CO}}(2 \rightarrow 1) / I_{\mathrm{CO}}(1 \rightarrow 0)=0.7$ (Leroy et al. 2012) and a standard Milky Way conversion factor of $\alpha_{\mathrm{CO}}=4.4 M_{\odot}$ $\mathrm{pc}^{-2}\left(\mathrm{~K} \mathrm{~km} \mathrm{~s}^{-1}\right)^{-1}$ equivalent to $X_{\mathrm{CO}}=2.0 \times 10^{20} \mathrm{~cm}^{-2}$ $\left(\mathrm{K} \mathrm{km} \mathrm{s}^{-1}\right)^{-1}$. This assumption may not be correct for the central kiloparsec region of KINGFISH galaxies, where $\alpha_{\mathrm{CO}}$ tends to be a factor of $\sim 2$ lower than the galaxy mean (Sandstrom et al. 2013). This should not be a problem for this study: our analysis only focus on predominantly atomic regions (see Section 4); therefore, we exclude the molecular-dominated 
central kiloparsec regions of KINGFISH galaxies. Note that Equation (2), as for $\mathrm{HI}$, includes a factor of 1.36 to account for helium and $\cos i$ to correct for inclination. HERACLES is sensitive to molecular gas mass surface densities down to a $3 \sigma$ level of $\Sigma_{\mathrm{mol}} \sim 4 M_{\odot} \mathrm{pc}^{-2}$.

\subsection{Additional Data}

In order to trace the obscured and un-obscured components of the star formation activity in our galaxies, we use a combination of the $24 \mu \mathrm{m}$ and $\mathrm{H} \alpha$ emission. The $24 \mu \mathrm{m}$ maps were drawn from the Spitzer Infrared Nearby Galaxy Survey (SINGS; Kennicutt et al. 2003). The $\mathrm{H} \alpha$ images were assembled and processed by Leroy et al. (2012) and come mainly from the SINGS and Local Volume Legacy (Dale et al. 2009) surveys, but are also retrieved from GOLDMine (Gavazzi et al. 2003) and the Palomar Las Campanas Atlas (Hoopes et al. 2001; Boselli et al. 2002; Knapen et al. 2004). The $\mathrm{H} \alpha$ data were corrected for Galactic extinction (Schlegel et al. 1998), foreground stars were masked (Muñoz-Mateos et al. 2009b) and the [N II] contribution was removed (Kennicutt et al. 2008, 2009). We also use Spitzer/IRAC SINGS $3.6 \mu \mathrm{m}$ maps to measure stellar mass surface densities (see the Appendix). Finally, we use Herschel PACS 70 and $160 \mu \mathrm{m}$ maps drawn from the photometric KINGFISH sample (Dale et al. 2012).

\subsection{Methods}

\subsubsection{Convolution of the Data to a Common Resolution}

We convolve all of our maps to have the same angular resolution. The final angular resolution varies from galaxy to galaxy because the H I map beam size can be higher or lower than the angular resolution of the $[\mathrm{CII}], \mathrm{CO}$, and PACS $160 \mu \mathrm{m}$ maps $\left(\approx 13^{\prime \prime}\right)$. The $\mathrm{H} \alpha, 24 \mu \mathrm{m}$ and PACS $70 \mu \mathrm{m}$ maps all have higher resolutions than the [C II] and $\mathrm{CO}$ data. The common angular resolutions to which all maps of a particular galaxy were convolved are listed in Table 1. After the convolution, the physical sizes of our regions have a median size of $\approx 0.8 \mathrm{kpc}$.

\subsubsection{Star Formation Rate Measurements}

We measure star formation rate surface densities $\left(\Sigma_{\mathrm{SFR}}\right)$ using a combination of the $\mathrm{H} \alpha$ and $24 \mu \mathrm{m}$ emission following the calibration by Calzetti et al. (2007) (Equation (8)), which is optimized for resolved regions rather than global galaxies (Kennicutt et al. 2009). This calibration adopts a truncated Salpeter IMF with a slope of 1.3 in the range of $0.1-0.5 M_{\odot}$ and a slope of 2.3 in the range of $0.5-120 M_{\odot}$.

\subsubsection{Dust-weighted Mean Starlight Intensity, $\langle U\rangle$, from the Draine and Li Dust Modeling}

The thermal pressure of the diffuse, neutral gas is proportional to the FUV radiation field strength (Wolfire et al. 2003). As we discuss in Section 6, the latter can be connected to the dust-weighted mean starlight intensity as calculated in the Draine \& Li (2007) model. In this model, dust is exposed to a range of radiation fields that give rise to two components: (1) the "Photodissociation region (PDR) component", where a fraction $\gamma$ of the dust mass is heated by a power-law distribution of intensities $U$ over a wide range, $U_{\min } \leqslant U \leqslant U_{\max }$ (and $U_{\max } \gg U_{\min }$ ); and (2) a "diffuse ISM" component, which is heated by a single ( $\delta$ function) radiation field, $U=U_{\min }$. This component contains most of the dust. The dust-weighted mean starlight intensity is defined as (Equation (17) in Draine \& Li 2007):

$$
\langle U\rangle=\left[(1-\gamma) U_{\min }+\frac{\gamma \ln \left(U_{\max } / U_{\min }\right)}{U_{\min }^{-1}-U_{\max }^{-1}}\right] .
$$

We estimate the dust-weighted mean starlight intensity, $\langle U\rangle$, using the 70-160 $\mu \mathrm{m}$ ratio and the empirical fit to the Draine \& Li (2007) model derived by Muñoz-Mateos et al. (2009a). $\langle U\rangle$ is normalized to the local interstellar radiation field measured by Mathis et al. (1983). This fit is only valid for regions with $\langle U\rangle \gtrsim 0.7$ given that below this value submillimeter data is needed to constrain the dust temperature of the source (Draine $\&$ Li 2007).

\section{3. [C II] $158 \mu \mathrm{m}$ Emission and the Thermal Pressure in the Diffuse, Neutral ISM}

The [C II] $158 \mu \mathrm{m}$ emission is the result of the radiative deexcitation of carbon ions $\left(\mathrm{C}^{+}\right)$collisionally excited by electrons $\left(\mathrm{e}^{-}\right)$, hydrogen atoms $(\mathrm{H})$, and/or hydrogen molecules $\left(\mathrm{H}_{2}\right)$. Which of these collisional partners dominate the excitation of $\mathrm{C}^{+}$depends on the properties of the gas where [C II] emission originates. Neutral carbon has a lower ionization potential $(11.3 \mathrm{eV})$ than hydrogen, thus ionized carbon can be found in both neutral (diffuse neutral gas and surface layers of PDRs) and ionized gas phases of the ISM. The goal of this section is to describe the method by which we can use the observed [C II] intensity and $\mathrm{H} \mathrm{I}$ column density to measure the volume density and thermal pressure of the neutral gas in the CNM phase. This requires the identification of the fraction of the observed [C II] intensity and the column density of $\mathrm{H}$ nuclei that is associated with the CNM. In this section, we go through this calculation by considering the multiphase origin of the [C II] emission, and the contribution to the column density of $\mathrm{H}$ nuclei by the CNM, the WNM, and the translucent part of clouds where $\mathrm{CO}$ has been dissociated.

In the optically thin limit, the [C II] integrated line intensity $I_{[\mathrm{C} \text { III }}$ resulting from the collisional excitation of $\mathrm{C}^{+}$by a given collisional partner in the neutral or ionized gas is given by (e.g., Crawford et al. 1985; Goldsmith et al. 2012)

$$
I_{[\mathrm{C} \mathrm{II}}=2.3 \times 10^{-21}\left(\frac{2 e^{-91.2 / T}}{1+2 e^{-91.2 / T}+\left(A_{\mathrm{ul}} / \sum_{i} R_{\mathrm{ul}, i} n_{i}\right)}\right) N_{\mathrm{C}^{+}} .
$$

Here, $I_{[\mathrm{C} \mathrm{I}]}$ is in units of $\mathrm{erg} \mathrm{s}^{-1} \mathrm{~cm}^{-2} \mathrm{sr}^{-1}, T$ is the kinetic temperature in $\mathrm{K}, N_{\mathrm{C}^{+}}$is the column density of $\mathrm{C}^{+}\left(\mathrm{in}^{-2}\right)$ in the $\mathrm{C}^{+}$region, $A_{\mathrm{ul}}$ is the Einstein spontaneous decay rate $\left(A_{\mathrm{ul}}=2.3 \times 10^{-6} \mathrm{~s}^{-1}\right), n$ is the volume density of the collisional partner, and $R_{\mathrm{ul}}$ is the collisional de-excitation rate coefficient at a kinetic temperature $T$ for a given collisional partner. The sum in the denominator is over collision partners (i.e., $\mathrm{H}, \mathrm{H}_{2}, \mathrm{He}$ or $\mathrm{e}^{-}$). To calculate the value of $R_{\mathrm{ul}}$ at a given $T$, we use the expressions in Goldsmith et al. (2012) for collisions with hydrogen atoms and electrons, and Wiesenfeld \& Goldsmith (2014) for collisions with hydrogen molecules.

The observed [C II] intensity, $I_{[\mathrm{C} \mathrm{II}}^{\mathrm{obs}}$, is the combination of the contributions to the $\left[\mathrm{C}\right.$ II] emission from the neutral gas $\left(I_{[\mathrm{C} \mathrm{II}]}^{\text {neutral }}\right)$ 
and the ionized gas $\left(I_{[\mathrm{C} \text { II }]}^{\text {ion }}\right)$, thus

$$
\left.I_{[\mathrm{C} \text { II }]}^{\text {obs }}=I_{[\mathrm{C} \text { II }]}^{\text {neutral }}+I_{[\mathrm{C}}^{\text {ion }}\right] \text {. }
$$

If we assume that the fraction of the observed [C II] intensity produced in the ionized gas is $f_{\text {ion }}$, then

$$
I_{[\mathrm{C} \text { II }]}^{\text {neutral }}=\left(1-f_{\text {ion }}\right) I_{[\mathrm{C} \mathrm{II}]}^{\mathrm{obs}} \text {. }
$$

For typical CNM and WNM conditions, we expect $I_{[\mathrm{C} \mathrm{II}}^{\mathrm{CNM}} / I_{[\mathrm{C} \mathrm{II}}^{\mathrm{WNM}} \approx 20^{15}$, so we can neglect the WNM contribution to the total $[\mathrm{C}$ II $]$ emission, i.e., $I_{[\mathrm{C} \mathrm{II}]}^{\text {neutral }} \approx I_{[\mathrm{C} \mathrm{II}]}^{\mathrm{CNM}}$.

Now, the $[\mathrm{C} \mathrm{II}]$ emission arising from the CNM is the result of collisional excitation by hydrogen atoms, molecules, He, and electrons. If we assume a typical ionization fraction for the CNM of $n_{\mathrm{e}} / n_{\mathrm{H}}=4.3 \times 10^{-4}$ (Draine 2011), then collisional excitations by electrons can be neglected. ${ }^{16}$ The same is true for collisional excitations of $\mathrm{C}^{+}$by He given that the He collision rate coefficient is only $\sim 4 \%$ of the $\mathrm{H}$ collision rate after assuming a cosmic abundance number ratio of $\mathrm{H} / \mathrm{He}=10$ (Draine 2011).

The CNM consists of the fraction $f_{\mathrm{CNM}}$ of the $\mathrm{HI}$, plus the diffuse, "CO-dark" $\mathrm{H}_{2}$. If $N_{\mathrm{H}_{2} \text {,diff }}$ is the column density of $\mathrm{H}_{2}$ in the diffuse gas and translucent part of clouds where $\mathrm{CO}$ has been dissociated (commonly referred as "CO-dark" or "COfaint" gas), then the total column density of $\mathrm{H}$ nuclei in the

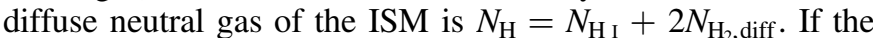
[C II] emission originates primarily from this component, then the $[\mathrm{C} \mathrm{II}]$ cooling rate per $\mathrm{H}$ nucleon is

$$
\Lambda_{[\mathrm{C} \mathrm{II}]}=\frac{4 \pi I_{[\mathrm{C} \mathrm{II}]}}{N_{\mathrm{H}}} .
$$

Let $f_{\mathrm{H}_{2} \text {, diff }}=2 N_{\mathrm{H}_{2} \text {, diff }} /\left(N_{\mathrm{H} \text { I }}+2 N_{\mathrm{H}_{2} \text {, diff }}\right)$ be the fraction of the diffuse ISM mass contributed by "CO-dark" $\mathrm{H}_{2}$. Then we can express $N_{\mathrm{H}}$ as $N_{\mathrm{H}}=N_{\mathrm{H} \text { I }} /\left(1-f_{\mathrm{H}_{2} \text {,diff }}\right)$ and write the [C II] cooling rate of the diffuse gas in the CNM as

$$
\Lambda_{[\mathrm{C} \text { II }]}^{\mathrm{CNM}}=\frac{4 \pi I_{[\mathrm{C} \mathrm{II}]}^{\mathrm{CNM}}}{f_{\mathrm{CNM}} N_{\mathrm{H} \mathrm{I}} /\left(1-f_{\mathrm{H}_{2} \text {,diff }}\right)} .
$$

Next, combining Equations (4)-(8), and assuming that the atomic and molecular hydrogen $\mathrm{C}^{+}$collisional rates are similar (Goldsmith et al. 2012; Wiesenfeld \& Goldsmith 2014), we link the observed [C II] cooling rate per $\mathrm{H}$ nucleon to the CNM neutral gas volume density $\left(n_{\mathrm{H}}+n_{\mathrm{H}_{2}}\right)$, resulting in

$$
\begin{aligned}
\Lambda_{[\mathrm{C} \mathrm{II}]}^{\mathrm{CNM}} \approx & 2.9 \times 10^{-20}\left(\frac{1-f_{\mathrm{H}_{2}, \text { diff }}}{f_{\mathrm{CNM}}}\right)\left(1-f_{\mathrm{ion}}\right)\left(\frac{\mathrm{C}}{\mathrm{H}}\right)_{\mathrm{gas}} \\
& \times\left(\frac{2 e^{-91.2 / T}}{1+2 e^{-91.2 / T}+\left(A_{\mathrm{ul}} / R_{\mathrm{ul}, \mathrm{H} \mathrm{I}}\right) /\left(n_{\mathrm{H} \mathrm{I}}+n_{\mathrm{H}_{2}}\right)}\right) .
\end{aligned}
$$

\footnotetext{
15 If we consider a CNM phase with $n_{\mathrm{H} \mathrm{I}}=50 \mathrm{~cm}^{-3}$ and $T=100 \mathrm{~K}$, a WNM phase with $n_{\mathrm{H}}=0.5 \mathrm{~cm}^{-3}$ and $T=8000 \mathrm{~K}$, and comparable column densities in the WNM and CNM, then $R_{u l, \mathrm{HI}}(T=100 \mathrm{~K})=7.6 \times 10^{-10} \mathrm{~cm}^{3} \mathrm{~s}^{-1}$, $R_{u l, \mathrm{HI}}(T=8000 \mathrm{~K})=1.4 \times 10^{-9} \mathrm{~cm}^{3} \mathrm{~s}^{-1}$ (Goldsmith et al. 2012), and $I_{[\mathrm{CII}]}^{\mathrm{CNM}} / I_{[\mathrm{C} \mathrm{II}]}^{\mathrm{WNM}} \approx\left(50 R_{u l, \mathrm{H} \mathrm{I}}(100) e^{-91.2 / 100}\right) /\left(0.5 R_{u l, \mathrm{H} \mathrm{I}}(8000) e^{-91.2 / 8000}\right) \approx 20$. ${ }^{16}$ If we assume for the CNM phase an ionization fraction $n_{\mathrm{e}} / n_{\mathrm{H}}=4.3 \times 10^{-4} \quad($ Draine 2011$)$ and $R_{u l, \mathrm{e}}(100 \mathrm{~K})=1.4 \times 10^{-9}$ $\mathrm{cm}^{3} \mathrm{~s}^{-1}$ (Goldsmith et al. 2012), then $I_{[\mathrm{C} \mathrm{II}]}^{\mathrm{HI}} / I_{[\mathrm{C} \mathrm{II}]}^{\mathrm{e}} \approx\left(R_{u l, \mathrm{H} \mathrm{I}}(100) / R_{u l, \mathrm{e}}\right.$ $(100)) \times\left(1 / 4.3 \times 10^{-4}\right) \approx 7$.
}

The units of $\Lambda_{[\mathrm{C} \text { II] }}^{\mathrm{CNM}}$ are erg s${ }^{-1} \mathrm{H}_{\text {nuclei }}{ }^{-1}$. Here we have also assumed that all gas-phase carbon is in a singly ionized state, thus $N_{\mathrm{C}^{+}} / N_{\mathrm{H} \mathrm{I}}=(\mathrm{C} / \mathrm{H})_{\text {gas }}$.

Finally, using Equation (9), we solve for the density of the neutral gas of the CNM, $n_{\mathrm{H}}+n_{\mathrm{H}_{2}}$, and then calculate the thermal pressure of the neutral gas following

$$
\mathrm{P}_{\mathrm{th}} / k\left[\mathrm{~K} \mathrm{~cm}^{-3}\right]=\left(n_{\mathrm{H} \mathrm{I}}+n_{\mathrm{H}_{2}}+n_{\mathrm{He}}\right) T,
$$

where $n_{\mathrm{He}}$ is the volume density of helium. For $f_{\mathrm{H}_{2}} \lesssim 0.5$, and assuming a cosmic abundance number ratio of $\mathrm{H} / \mathrm{He}=10$, we can approximate $n_{\mathrm{H}}+n_{\mathrm{H}_{2}}+n_{\mathrm{He}} \approx$ $1.1\left(n_{\mathrm{H} \text { I }}+n_{\mathrm{H}_{2}}\right)$.

How sensitive is this $\Lambda_{[\mathrm{C} \text { II }}$-based thermal pressure measurement to changes in the assumptions of $T, f_{\mathrm{CNM}}, f_{\text {ion }}$, and $f_{\mathrm{H}_{2} \text {, diff }}$ ? Figure 1 shows the thermal pressure $P_{\mathrm{th}}$ as a function of the cooling rate $\Lambda_{[\mathrm{C} \text { II] }}$ for different assumptions on $T$ (panel 1), $f_{\mathrm{CNM}}$ (panel 2), and $f_{\text {ion }}$ and $f_{\mathrm{H}_{2} \text {, diff }}$ (panel 3). If we only vary the temperature of the $\mathrm{CNM}$ gas, we find that the resulting thermal pressure varies by about a factor of $\sim 1.5$ in the $40 \gtrsim T \gtrsim 100 \mathrm{~K}$ range, and about a factor of $\sim 2$ in the $40 \gtrsim T \gtrsim 400 \mathrm{~K}$ range. We conclude that [C II]-based thermal pressure measurements are very robust to even large variations of the CNM temperature. In the case of the CNM fraction, for a given $\Lambda_{[\mathrm{C} \text { II] }}$ (at fixed $T$ ) the thermal pressure increases by a factor of $\sim 2.3$ if we decrease the CNM fraction from $f_{\mathrm{CNM}}=0.7$ to $f_{\mathrm{CNM}}=0.3$. This change in the CNM fraction is expected if we go from the inner to the outer parts of the Galactic plane (Pineda et al. 2013). Finally, the third panel in Figure 1 shows that for a given $\Lambda_{[\mathrm{C} \text { II] }}$ (for fixed assumptions on $f_{\mathrm{CNM}}$ and $T$ ) the resulting thermal pressure decreases by a factor of $\sim 2$ if we increase the contribution from both, $f_{\text {ion }}$ and $f_{\mathrm{H}_{2}}$, diff , from $0 \%$ to $30 \%$.

\section{Measuring $\Lambda_{[\mathrm{C} \text { II }]}$ and $P_{\mathrm{th}}$ in the Kingfish Sample}

Calculating the thermal pressure of the CNM gas based on the $[\mathrm{CII}]$ emission requires selection of regions where the collisional excitation of $\mathrm{C}^{+}$is dominated by the diffuse, neutral gas component. In addition, a number of assumptions need to be made regarding the origin of the [C II] emission and the temperature and carbon abundance of the CNM gas (see Equation (9)). In this section, we describe our selection criteria for the KINGFISH regions and the assumptions underlying the thermal pressure calculation.

\subsection{Selection of Regions}

As a first step to build a sample of quiescent, neutral gas dominated regions, we start by identifying those where the mass surface density of atomic gas is higher than that of molecular gas.

Figure 2 shows the [C II] cooling rate as a function of the molecular ratio, $R_{\mathrm{mol}}=\Sigma_{\mathrm{mol}} / \Sigma_{\mathrm{H}}$, for 2093 regions for which we have $[\mathrm{C}$ II] and $\mathrm{HI}$ detections with $\mathrm{S} / \mathrm{N} \geqslant 3$. Triangles in the right panel represent regions for which we only have $3 \sigma$ upper limits on $\Sigma_{\text {mol }}$ driven by a non-detection in CO. In this particular case $\Lambda_{[\mathrm{C} \text { II] }}$ is calculated using Equation (9) assuming $f_{\text {ion }}=f_{\mathrm{H}_{2} \text {,diff }}=0$ and $f_{\mathrm{CNM}}=0.5$, i.e., half of the atomic gas is in the CNM phase (Heiles \& Troland 2003; Pineda et al. 2013). The color scale indicates the star formation rate surface density. We find that the [C II] cooling rate increases as a function of the molecular ratio, and that for a given $R_{\text {mol }}$, regions with higher $\Sigma_{\text {SFR }}$ have higher [C II] cooling rates. This 

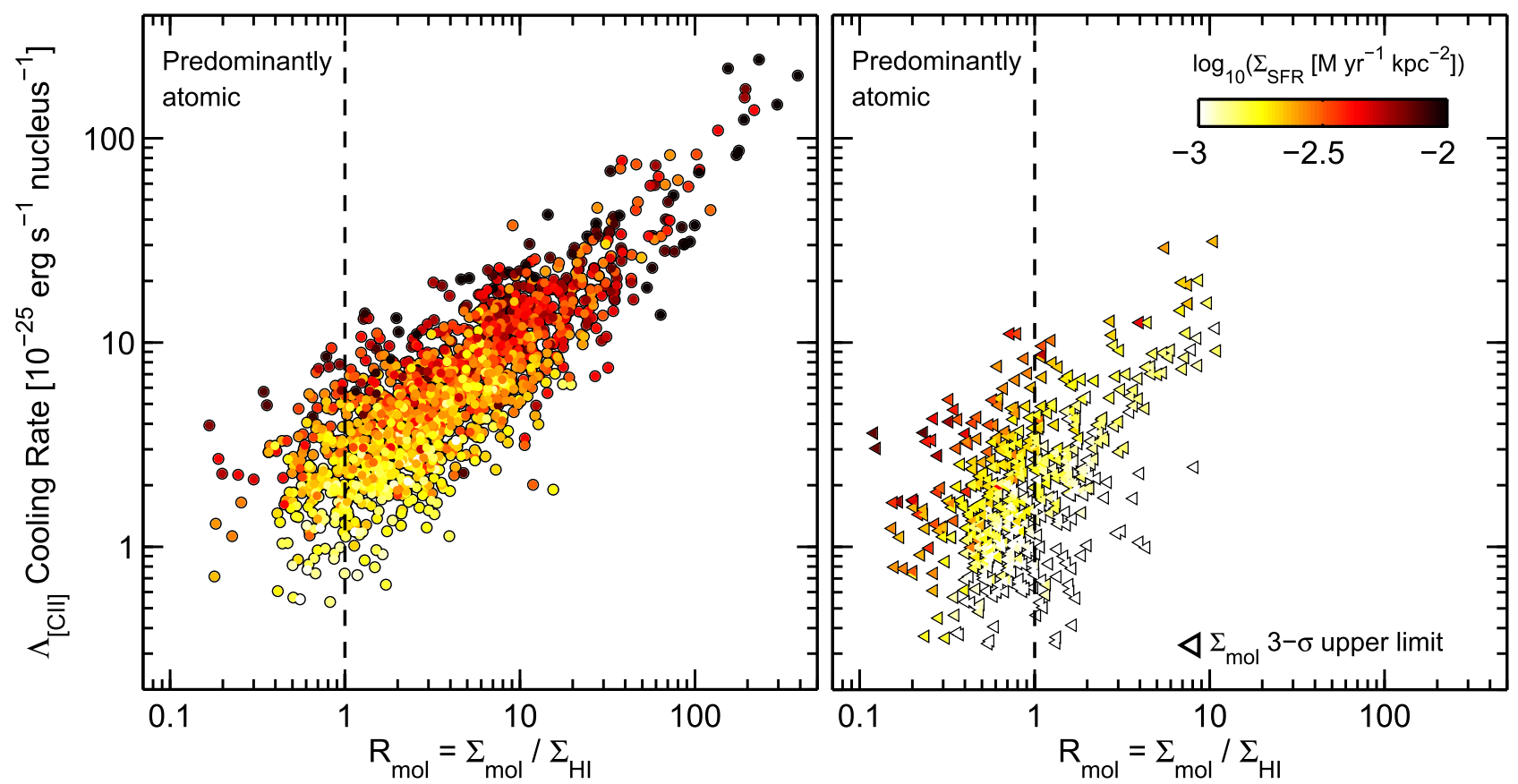

Figure 2. [C II] cooling rate per hydrogen nucleus $\left(\Lambda_{[\mathrm{C} \mathrm{II}}\right)$ as a function of $R_{\mathrm{mol}}$, the ratio between the surface density of atomic $\left(\Sigma_{\mathrm{H} \mathrm{I}}\right)$ and molecular $\left(\Sigma_{\mathrm{mol}}\right)$ gas. The latter employs a CO-based determination of the molecule content, and by definition does not include "CO-dark" gas. $\Lambda_{[\mathrm{C} \text { II] }}$ was calculated using Equation (9) and assuming $T=100 \mathrm{~K}, f_{\mathrm{CNM}}=0.5, f_{\mathrm{H} \text {,diff }}=f_{\text {ion }}=0$. The left panel shows the regions for which we have detections of [C II], CO, and $\mathrm{H} \mathrm{I}$ emission with $\mathrm{S} / \mathrm{N}>3$. The right panel, on the other hand, shows the regions for which we only have upper limits driven by non-detections in CO emission. We consider as predominantly atomic regions those with $R_{\mathrm{mol}} \leqslant 1$ (dashed line). In these regions, we expect the $\mathrm{C}^{+}$collisional excitations to be dominated by collisions with $\mathrm{H}$ atoms. The color scale represents the star formation rate surface density $\left(\Sigma_{\mathrm{SFR}}\right)$ of the regions measured as a combination of $24 \mu \mathrm{m}$ and $\mathrm{H} \alpha$ emission. Regions with lower [C II] cooling rates tend to show lower $\Sigma_{\mathrm{SFR}}$ values. This is expected given that in thermal equilibrium, the heating proportional to $\Sigma_{\mathrm{SFR}}$-and the cooling dominated by the [C II] emission-are in balance.

is expected in thermal equilibrium, where the heating of the gas powered by the star formation activity is balanced by the cooling, of which the [C II] emission is one of the main channels (e.g., Herrera-Camus et al. 2015).

From a total of 2093 regions with [C II], CO, and $\mathrm{HI}$ data available, we select for this study of the thermal pressure of the neutral gas 534 regions with $R_{\mathrm{mol}} \leqslant 1$ (345 of these regions have upper limits on $\Sigma_{\mathrm{mol}}$ ). Lowering the cut-off level to $R_{\mathrm{mol}} \leqslant 0.5$ reduces the number of selected regions to 145 , but it does not have a significant effect on the distribution of [C II] cooling rates.

\subsection{Assumptions Made in the $P_{\text {th }}$ Calculation}

As Equation (9) shows, to measure the thermal pressure of the CNM gas we need to make assumptions about the temperature of the CNM gas, the carbon abundance, the fraction of the atomic gas in the CNM, the contribution to the [C II] emission from the ionized gas, and the mass fraction of diffuse molecular gas mixed with the atomic gas.

CNM temperature: In this work, we assume a CNM temperature of $T=100 \mathrm{~K}$ (Gerin et al. 2015). Remember that a change in the temperature assumption of a factor of $\sim 2$ around $T=100 \mathrm{~K}$ will only have a small effect $(\lesssim 30 \%)$ on the determination of $P_{\text {th }}$ (see Figure 1).

Carbon abundance: To determine the gas-phase carbon abundance of a particular region, we use as a proxy the oxygen abundance of its parent galaxy. Characteristic gas phase oxygen abundances in $\mathrm{H}$ II regions of our galaxies were measured by Moustakas et al. (2010) based on the theoretical KK04 and empirical PT05 calibration methods. The latter yields metallicities that are systematically lower by about $\sim 0.6$ dex compared to those obtained using the KK04 calibration; together, they represent the full range of metallicities one would obtain using other strong-line abundance calibrations (Moustakas et al. 2010). For the two calibration methods, we convert the $\mathrm{O}$ abundances into diffuse depleted carbon abundances using the analytic function in the latest version of the MAPPINGS photoionization code (Nicholls et al. 2016), i.e.,

$$
\log (\mathrm{C} / \mathrm{H})=\log (\mathrm{O} / \mathrm{H})+\log \left(10^{-1.00}+10^{(2.72+\log (\mathrm{O} / \mathrm{H}))}\right) .
$$

This analytic function has been re-normalized so that if we input the oxygen gas-phase abundance measured in the Orion nebula $\quad(12+\log (\mathrm{O} / \mathrm{H})=8.65 ; \quad$ Simón-Díaz \& $\quad$ Stasińska 2011), we recover a local Galactic depleted ISM carbon abundance of $(\mathrm{C} / \mathrm{H})=1.5 \times 10^{-4}$ (Gerin et al. 2015). We take as the final carbon abundance the average between the two carbon abundances derived independently from the KK04 and PT05 oxygen metallicities following Equation (11).

Fraction of the atomic gas in the CNM $\left(f_{\mathrm{CNM}}\right)$ : Following the results from Heiles \& Troland (2003) and Pineda et al. (2013) we assume $f_{\mathrm{CNM}}=0.5$ (i.e., half of the atomic gas is in the CNM phase), but we also allow this fraction to vary in the $0.3-0.7$ range.

Contribution to the [C II] emission from ionized gas $\left(f_{\text {ion }}\right)$ : There is a fraction of the observed [C II] emission that is the result of collisional excitations in $\mathrm{HII}$ gas. For a proper calculation of the $P_{\text {th }}$ in the neutral gas, this additional contribution needs to be subtracted. One method to account for the contribution from the ionized gas is to use the [N II] $205 \mu \mathrm{m}$ transition. Given that this line arises exclusively 

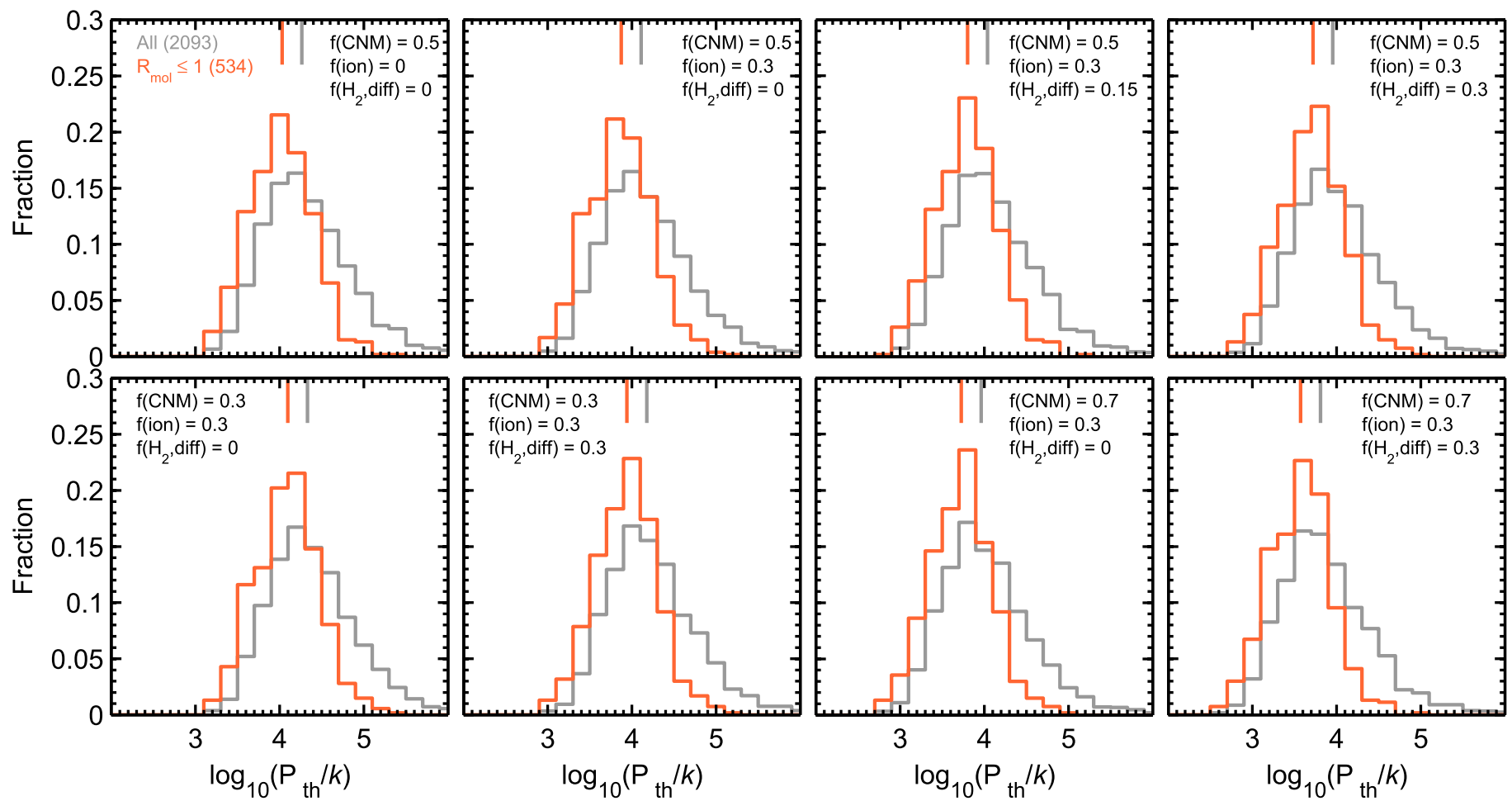

Figure 3. Thermal pressure distribution for all regions (gray histograms) and atomic-dominated regions (orange histograms) selected from the KINGFISH sample. Each panel shows the resulting distributions after assuming different values for the fraction of neutral atomic gas in the CNM $\left(f_{\mathrm{CNM}}\right)$, and the contribution to the [C II] emission from collisional excitations by electrons $\left(f_{\text {ion }}\right)$ and diffuse molecular hydrogen gas $\left(f_{\mathrm{H}_{2} \text {,diff }}\right)$. (Panels 1-4) We assume $f_{\mathrm{CNM}}=0.5$ and $f_{\text {ion }}=0.3$ and we increase the value of $f_{\mathrm{H}_{2} \text {,diff }}$ from 0 to 0.3. (Panels 5-6) We assume $f_{\mathrm{CNM}}=0.7$ and $f_{\text {ion }}=0.3$ and we increase the value of $f_{\mathrm{H}_{2} \text {,diff }}$ from 0 to 0.3 . For a fixed CNM temperature and carbon abundance, the net effect of increasing $f_{\mathrm{CNM}}, f_{\text {ion }}$ and/or $f_{\mathrm{H}_{2} \text {,diff }}$ is to reduce $\Lambda_{[\mathrm{C} \text { II] }}^{\mathrm{CNM}}$ (Equation (9)). This implies a decrease in the neutral gas density, and as a result, a decrease in the thermal pressure.

from the ionized gas and has a critical density similar to that of the [C II] line in the ionized medium, the [C II] to [N II] $205 \mu \mathrm{m}$ ratio is a tracer of the fraction of [C II] emission that originates in the ionized gas, $f_{\text {ion. }}$. Based on [N II] $205 \mu \mathrm{m}$ observations from the "Beyond the Peak" survey-that include multiple regions selected from 21 of the galaxies in our sample- $f_{\text {ion }}$ is estimated to be $f_{\text {ion }} \sim 0.2-0.4$ for regions with infrared colors $\nu f_{\nu}(70) / \nu f_{\nu}(160) \lesssim 1.5$, and $f_{\text {ion }} \sim 0.1-0.15$ for warmer regions with infrared colors between $1.5 \lesssim \nu f_{\nu}(70) / \nu f_{\nu}$ $(160) \lesssim 2.5$ (K. Croxall et al. 2017, in preparation). In our sample of predominantly atomic regions, $92 \%$ have $\nu f_{\nu}(70) / \nu f_{\nu}(160) \lesssim 1.5$, and thus we fix the contribution from the ionized gas to the total [C II] emission to be $f_{\text {ion }}=0.3$. This is consistent with the ionized gas contribution to the total [C II] luminosity of the Milky Way measured by Pineda et al. (2014).

Mass fraction of diffuse $\mathrm{H}_{2}$ gas mixed with the atomic gas $\left(f_{\mathrm{H}, \text { diff }}\right)$ : Observations and modeling suggest that $30 \%-50 \%$ of diffuse $\mathrm{H}_{2}$ in the Solar Neighborhood resides in a "CO-dark" phase (Grenier et al. 2005; Wolfire et al. 2010). In the Galactic plane, Langer et al. (2014) find a range of mass fractions of "CO-dark" $\mathrm{H}_{2}$ in molecular clouds that goes from $\sim 20 \%$ in dense clouds to $\sim 75 \%$ in diffuse molecular clouds. Molecular hydrogen in this "CO-dark" gas can contribute to the [C II] emission by collisionally exciting $\mathrm{C}^{+}$ions with a collisional rate roughly similar to that of hydrogen atoms (Goldsmith et al. 2012; Wiesenfeld \& Goldsmith 2014). Unfortunately, with our current data set, it is very difficult to constrain the amount of "CO-dark" $\mathrm{H}_{2}$ gas mixed with the atomic $\mathrm{H}$ I gas. The only option we are left with is to correct the [C II] cooling rate by assuming a mass fraction of diffuse $\mathrm{H}_{2}$ gas that is not traced by $\mathrm{CO}$. In this work, and motivated by the observational and modeling results described above, we present results for thermal pressures calculated assuming diffuse $\mathrm{H}_{2}$ mass fractions of $f_{\mathrm{H}_{2} \text {, diff }}=0,0.15$, and 0.3 .

\section{Distribution of Thermal Pressures}

The thermal pressure distributions resulting from assuming different values of $f_{\mathrm{CNM}}, f_{\text {ion }}$, and $f_{\mathrm{H}_{2} \text {,diff }}$ are presented in Figure 3. Each panel shows the results for all regions initially considered for this study (2093 in total), and the 534 regions with molecular ratios $R_{\mathrm{mol}} \leqslant 1$. We note that $R_{\mathrm{mol}}$ employs a CO-based determination of the molecule content, and by definition does not include "CO-dark" gas. In the upper panels of Figure 3, we assume that $f_{\mathrm{CNM}}=0.5$, and then from left to right we show the resulting thermal pressure distributions if we increase $f_{\text {ion }}$ and $f_{\mathrm{H}_{2} \text {,diff }}$ contributions from $0 \%$ to $30 \%$. The net effect of increasing $f_{\text {ion }}$ and $f_{\mathrm{H}_{2} \text {, diff }}$ is that the thermal pressure decreases by a factor of $\sim 2$. The bottom panels show the effect on the thermal pressure distributions if we now assume CNM fractions of $f_{\mathrm{CNM}}=0.3$ and $f_{\mathrm{CNM}}=0.7$ instead of $f_{\mathrm{CNM}}=0.5$. We observe that the median $P_{\mathrm{th}}$ in the predominately atomic regions, when we assume $f_{\mathrm{CNM}}=0.3$, is a factor of $\sim 2.3$ lower than when we assume $f_{\mathrm{CNM}}=0.7$. The decrease in the thermal pressure with increasing $f_{\text {ion }}, f_{\mathrm{CNM}}$, and/or $f_{\mathrm{H}_{2} \text {,diff }}$ fractions is expected from Equation (9). The [C II] cooling rate per $\mathrm{H}$ nucleon of the diffuse $\mathrm{CNM}\left(\Lambda_{[\mathrm{C} \text { II] }}^{\mathrm{CNN}}\right)$ is proportional to $I_{[\mathrm{C} \text { II] }}^{\mathrm{CNM}} / N_{\mathrm{H}}$. Therefore, reducing $I_{[\mathrm{C} \mathrm{II}]}^{\mathrm{CNM}}$ by increasing $f_{\text {ion }}$, or increasing $N_{\mathrm{H}}$ by increasing $f_{\mathrm{CNM}}$ and/or $f_{\mathrm{H}_{2} \text {,diff }}$, will result in a 


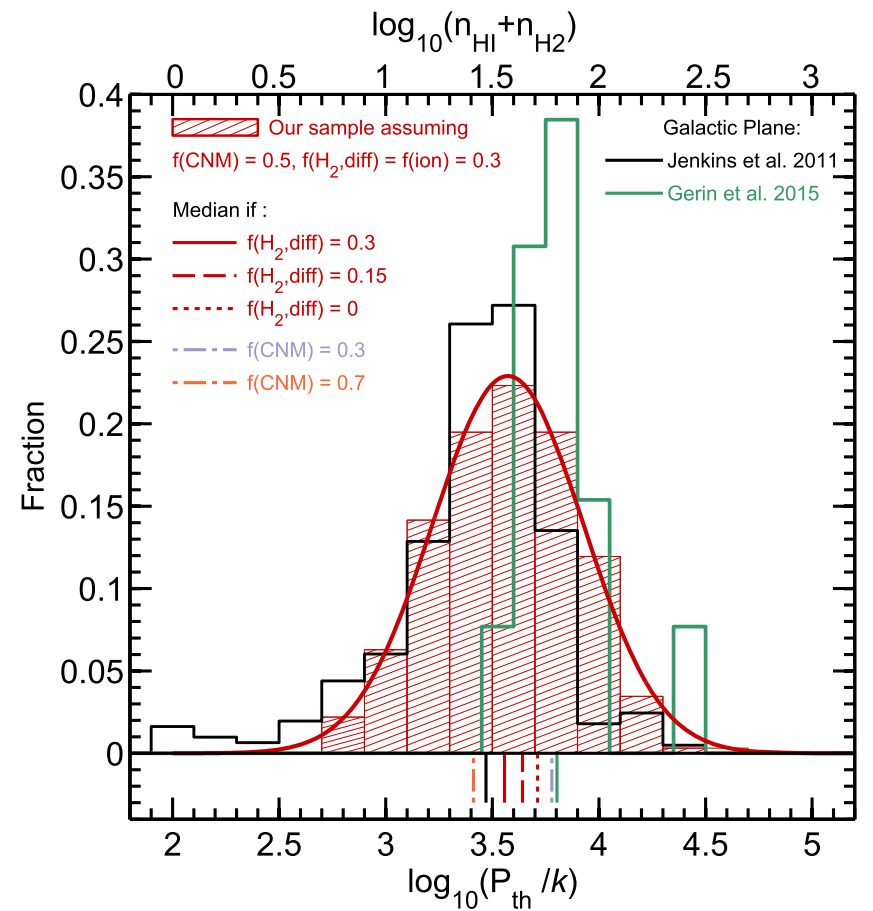

Figure 4. Comparison between the thermal distributions from a sub-sample of KINGFISH regions (318 regions with $\langle U\rangle \leqslant 3$ and $R_{\text {mol }} \leqslant 1$; red histogram) and regions in the Galactic plane from Jenkins \& Tripp (2011; 614 regions, black histogram) and Gerin et al. (2015; 13 regions, green histogram). The corresponding value of the diffuse, neutral gas density $n_{\mathrm{HI}}+n_{\mathrm{H}_{2}}$ is shown in the upper axis. For the thermal pressure calculations involving the KINGFISH regions, we have assumed $f_{\mathrm{CNM}}=0.5, f_{\text {ion }}=0.3$, and $f_{\mathrm{H}_{2} \text {,diff }}=0.3$. The KINGFISH distribution of $P_{\text {th }}$ can be well represented by the log-normal distribution described in Equation (12). In the bottom of the figure, we show the median thermal pressure measured in our sample when assuming $f_{\mathrm{CNM}}=0.5, f_{\text {ion }}=0.3$, and $f_{\mathrm{H}_{2} \text {, diff }}=0.3$ (red solid line), $f_{\mathrm{H}_{2} \text {,diff }}=0.15$ (red dashed line) or $f_{\mathrm{H}_{2} \text {, diff }}=0$ (red dotted line). We also include the median values when we vary the $\mathrm{CNM}$ fraction, i.e., we assume $f_{\text {ion }}=f_{\mathrm{H}_{2} \text {,diff }}=0.3$ and $f_{\mathrm{CNM}}=0.3$ (purple line) or $f_{\mathrm{CNM}}=0.7$ (orange line). The median thermal pressures measured in Jenkins \& Tripp (2011) and Gerin et al. (2015) samples are shown in black and green, respectively.

lower value of $\Lambda_{[\mathrm{C} \text { II] }}^{\mathrm{CNM}}$. In the low density limit $\left(n \ll n_{\text {crit }}\right)$, and for a given CNM temperature and carbon abundance, the cooling rate is $\Lambda_{[\mathrm{C} I]}^{\mathrm{CNM}} \propto\left(n_{\mathrm{H}_{\mathrm{I}}}+n_{\mathrm{H}_{2}}\right) \propto P_{\mathrm{th}}$, so the thermal pressure increases or decreases at the same rate as the [C II] cooling rate.

Given that we expect $f_{\text {ion }}$ to be close to 0.3 (K. Croxall et al. 2017, in preparation), and that individual changes in the assumption of $f_{\mathrm{H}_{2} \text {, diff }}$ produce changes that are $\lesssim 30 \%$, we expect that the comparison of our results to measurements in the Galactic plane and expectations from models can help us to constrain $f_{\mathrm{CNM}}$.

\subsection{Comparison to the Galactic Distribution of Thermal Pressures}

We compare the distribution of thermal pressures in our sample of predominantly atomic regions to those observed in the diffuse gas of the Galactic plane (Jenkins \& Tripp 2011; Goldsmith 2013; Gerin et al. 2015). In the case of Jenkins \& Tripp (2011), they select regions in some measure removed from the influence of bright stars by excluding those where the starlight intensity is $>3$ times the Galactic average. We impose a similar condition based on $\langle U\rangle$ to the 534 regions with $R_{\text {mol }} \leqslant 1$, resulting in a sub-sample of 318 quiescent, predominantly atomic regions. Figure 4 shows the distribution of thermal pressures for this sub-sample when assuming $f_{\mathrm{CNM}}=0.5, f_{\text {ion }}=0.3$ and $f_{\mathrm{H}_{2} \text {, diff }}=0.3$. The distribution can be well represented by a log-normal distribution given by

$$
f\left(P_{\mathrm{th}} / k\right)=0.23 \times \exp \left(-\frac{\left(\log _{10}\left(P_{\mathrm{th}} / k\right)-3.57\right)^{2}}{2(0.35)^{2}}\right) .
$$

We measure a median thermal pressure of $P_{\mathrm{th}} /$ $k=3610 \mathrm{~K} \mathrm{~cm}^{-3}$. Compared to the thermal pressures measured in the Galactic plane, our result is similar to the mean value calculated by Jenkins \& Tripp (2011), and about 50\% lower than the median thermal pressure values calculated by Goldsmith (2013) and Gerin et al. (2015). The bottom part of Figure 4 expands this comparison by including the value of the median thermal pressure in our sample for different assumptions on $f_{\mathrm{H}_{2} \text {,diff }}$ and $f_{\mathrm{CNM}}$. We note that we find a better agreement with the Gerin et al. (2015) results if we assume $f_{\mathrm{H}_{2} \text {, diff }} \leqslant 0.15$ or $f_{\mathrm{CNM}}=0.3$. In addition, we can obtain a median thermal pressure value slightly closer to the one measured by Jenkins \& Tripp (2011) if we increase the CNM fraction to $f_{\mathrm{CNM}}=0.7$. The latter case is less likely given that CNM fractions greater than $\sim 0.5$ are probably too high for diffuse gas. On the other hand, if we assume $f_{\mathrm{CNM}}=0.3$ the resulting median thermal pressure is $P_{\mathrm{th}} / k=8100 \mathrm{~K} \mathrm{~cm}^{-3}$, almost a factor three higher than the median thermal pressure measured by Jenkins \& Tripp (2011). Overall, the comparison between our sample and the Galactic plane results favors ISM properties on $\sim$ kiloparsec scales, where $f_{\mathrm{CNM}} \sim 0.5$ and $0.15 \lesssim f_{\mathrm{H}_{2} \text {, diff }} \lesssim 0.3$.

\section{Thermal Pressure and Star Formation Activity}

In the two-phase model for the ISM, the CNM and WNM phases can coexist in pressure equilibrium when the thermal pressure lies within a range set by $P_{\min }$ and $P_{\max }$. We define the two-phase pressure as the geometric mean between these two, i.e., $P_{\text {two-phase }}=\left(P_{\min } P_{\max }\right)^{1 / 2}$. Hydrodynamical simulations with self-consistent gravitational collapse and star formation feedback to heat and drive turbulence in the ISM find the thermal pressure of the multiphase medium to be close to the $P_{\text {two-phase }}$ pressure (Kim et al. 2011, 2013).

Based on the definition of $P_{\text {two-phase }}$ and using the expression for $P_{\min }$ derived by Wolfire et al. (2003), we can express $P_{\text {two-phase }}$ as

$$
\begin{aligned}
\frac{P_{\text {two-phase }}}{k} \simeq & 8500 \mathrm{~K} \mathrm{~cm}^{-3}\left(\frac{P_{\max }}{P_{\min }}\right)^{1 / 2} \\
& \times \frac{G_{0}^{\prime} Z_{d}^{\prime} / Z_{g}^{\prime}}{1+3.1\left(G_{0}^{\prime} Z_{d}^{\prime} / \zeta_{t}^{\prime}\right)^{0.365}} .
\end{aligned}
$$

Here, pressure is defined in units of $\mathrm{K} \mathrm{cm}^{-3}, G_{0}^{\prime}$ corresponds to the mean FUV intensity relative to the value measured locally $\left(I_{\mathrm{FUV}, 0}=2.1 \times 10^{-4} \mathrm{erg} \mathrm{cm}^{-2} \mathrm{~s}^{-1} \mathrm{sr}^{-1}\right.$; Draine 1978), $Z_{d}^{\prime}$ and $Z_{g}^{\prime}$ are the dust and gas abundances relative to the solar neighborhood values, and $\zeta_{t}^{\prime}$ corresponds to the total cosmicray $/ \mathrm{EUV} / \mathrm{X}$-ray ionization rate relative to the value $10^{-16} \mathrm{~s}^{-1}$. The ratio between $P_{\min }$ and $P_{\max }$ depends on various properties of the gas, including metallicity and the shielding of ionizing 
radiation. According to Wolfire et al. (1995, 2003), we expect $P_{\max } / P_{\min }$ to be in the $\sim 2-5$ range.

In order to compare our results to the model predictions, we rewrite Equation (13) in terms of available observational quantities. Following a procedure similar to that of Ostriker et al. (2010), we first express $G_{0}^{\prime}$ as $G_{0}^{\prime}=I_{\mathrm{FUV}} / I_{\mathrm{FUV}, 0} \approx$ $\Sigma_{\mathrm{SFR}} / \Sigma_{\mathrm{SFR}, 0}$, where $\Sigma_{\mathrm{SFR}, 0}=2.5 \times 10^{-3} M_{\odot} \mathrm{yr}^{-1} \mathrm{kpc}^{-2}$ is the star formation rate surface density in the solar neighborhood (Fuchs et al. 2009). Then, we assume that the total cosmic-ray/ $\mathrm{EUV} / \mathrm{X}$-ray ionization rate $\zeta_{t}^{\prime}$ is proportional to $\Sigma_{\mathrm{SFR}}$ and inversely proportional to the total gas surface density $\Sigma_{\text {gas }}=\Sigma_{\mathrm{H}_{\mathrm{I}}}+\Sigma_{\mathrm{H}_{2}}$. This assumption is discussed in detail in Wolfire et al. (2003), and originates from the fact that cosmicrays and the hot gas that produces the X-ray emission are related to supernova explosions, while the opacity is related to the surface density of the neutral gas. Thus, $G_{0}^{\prime} / \zeta_{t}^{\prime}=\Sigma_{\text {gas }} / \Sigma_{\text {gas, } 0}$, where $\Sigma_{\text {gas }, 0}$ is the surface gas density in the solar neighborhood. For our calculations, we assume $\Sigma_{\text {gas,0 }}=10 \quad M_{\odot} \mathrm{kpc}^{-2}$ (Wolfire et al. 2003; Kalberla \& Kerp 2009). Finally, we assume that the dust-to-gas ratio follows the metallicity, i.e., $Z_{d}^{\prime} / Z_{g}^{\prime} \approx 1$. The new expression for $P_{\text {two-phase }}$ as a function of $\Sigma_{\mathrm{SFR}}$ is

$$
\begin{aligned}
& \frac{P_{\text {two-phase }}}{k} \simeq 3.5 \times 10^{6} \mathrm{~K} \mathrm{~cm}^{-3}\left(\frac{P_{\max }}{P_{\text {min }}}\right)^{1 / 2} \\
& \times \frac{\Sigma_{\mathrm{SFR}}}{M_{\odot} \mathrm{yr}^{-1} \mathrm{kpc}^{-2}} \times \frac{1}{1+3.1\left(Z_{d}^{\prime} \Sigma_{\text {gas }} / \Sigma_{\text {gas }, 0}\right)^{0.365}} .
\end{aligned}
$$

The units of $\Sigma_{\mathrm{SFR}}$ are $M_{\odot} \mathrm{yr}^{-1} \mathrm{kpc}^{-2}$ and pressure is defined in units of $\mathrm{K} \mathrm{cm}^{-3}$.

We derive a third expression for $P_{\text {two-phase, this time }}$ expressing $G_{0}$ in terms of the dust-weighted, mean starlight intensity $\langle U\rangle$ (see Section 2.5.3). The Mathis et al. (1983) field integrated between 6 and $13.6 \mathrm{eV}$ is related to the Habing field by a factor of 1.13 , so $G_{0}=1.13 U$. In the Draine \& Li (2007) model, the diffuse ISM is exposed to a single radiation field $U=U_{\min }$, while a fraction $\gamma$ of the dust mass is heated by a power-law distribution of intensities $U$ over the range of $U_{\min } \leqslant U \leqslant U_{\max }$ (where $U_{\max } \gg U_{\min }$ ). In our sample of predominately atomic regions, $\gamma$ is $\lesssim 0.1$ (Draine et al. 2007), which implies that the total power radiated by dust is dominated by dust in the diffuse component of the ISM exposed to $\langle U\rangle \approx U_{\min } \approx G_{0}$. Based on this, we rewrite

$$
\begin{aligned}
\frac{P_{\mathrm{two} \text {-phase }}}{k} \approx & 9600 \mathrm{~K} \mathrm{~cm}^{-3}\left(\frac{P_{\mathrm{max}}}{P_{\min }}\right)^{1 / 2} \\
& \times \frac{U_{\mathrm{min}}}{1+3.1\left(Z_{d}^{\prime} \Sigma_{\mathrm{gas}} / \Sigma_{\mathrm{gas}, 0}\right)^{0.365}} .
\end{aligned}
$$

In a two-phase ISM model in equilibrium, we expect $P_{\text {min }} \lesssim P_{\text {th }} \lesssim P_{\text {max }}$, with $P_{\text {th }}$ close to the value of $P_{\text {two-phase. In }}$ Figure 5, we explore these model expectations by comparing the thermal pressures of our sample of regions with $R_{\text {mol }} \leqslant 1$ (assuming $f_{\mathrm{H}_{2} \text {,diff }}=0.3$ ) to the predictions from the Wolfire et al. (2003) model. In the left panels, we show the correlation between $P_{\mathrm{th}}$ and $\Sigma_{\mathrm{SFR}}$ (upper panel), and $P_{\mathrm{th}}$ and $\langle U\rangle$ (lower panel). In both cases, we use color to indicate the value of $\Sigma_{\text {gas }}$. We include the model results from Equation (14) for the $P_{\mathrm{th}}-\Sigma_{\mathrm{SFR}}$ case, and Equation (15) for the $P_{\mathrm{th}}-\langle U\rangle$ correlation. In both cases, we assume $P_{\max } / P_{\min }=2, Z_{d}^{\prime}=1$ and three different values for the gas mass surface density, $\Sigma_{\text {gas }}=3$ (solid line), 10 (dashed line) and $20 M_{\odot} \mathrm{pc}^{-2}$ (dotted line). The hatched gray regions represent the maximal area where the condition $P_{\min } \lesssim P_{\text {th }} \lesssim P_{\max }$ is satisfied (for $2 \leqslant$ $P_{\max } / P_{\min } \leqslant 5,3 \leqslant \Sigma_{\text {gas }} /\left(M_{\odot} \mathrm{yr}^{-1}\right) \leqslant 20$ and $\left.0.3 \leqslant Z_{d}^{\prime} \leqslant 3\right)$. Finally, the red hatched regions mark where $\Sigma_{\mathrm{SFR}} \leqslant 10^{-3} M_{\odot}$ $\mathrm{yr}^{-1} \mathrm{kpc}^{-2}$ or $\langle U\rangle \leqslant 0.7$, our lower limits for a reliable $\Sigma_{\mathrm{SFR}}$ or $\langle U\rangle$ measurements, respectively.

As predicted by the model, we observe a correlation of increasing $P_{\text {th }}$ with increasing $\langle U\rangle$ (Spearman correlation coefficient $\rho=0.53$ ) and increasing $\Sigma_{\mathrm{SFR}}$ (Spearman correlation coefficient $\rho=0.60)$. This is expected due to the increase of the photoelectric heating as $G_{0}$, which is proportional to $\langle U\rangle$ and $\Sigma_{\mathrm{SFR}}$, rises.

\subsection{Dependence with Gas Mass Surface Density $\Sigma_{\text {gas }}$}

The Wolfire et al. (2003) model predicts that for a fixed SFR, the thermal pressure should decrease as a function of $\Sigma_{\text {gas }}$. Recall that $\zeta_{t}^{\prime} \propto\left(\Sigma_{\text {gas }}\right)^{-1}$, so if $\Sigma_{\text {gas }}$ drops, the electron abundance in the gas rises, which helps to neutralize the charge of the dust grains and thus increase the grain photoelectric heating efficiency. As can be seen from the position of the lines in the left panels of Figure 5, our data are also consistent with this prediction, as we observe that for a fixed $\Sigma_{\text {SFR }}$, regions with higher values of $\Sigma_{\text {gas }}$ tend to have lower thermal pressures. Finally, it is worth noting that the dispersion in our data $(\sim 0.35 \mathrm{dex})$ is larger than expected from the model, even if we assume the more extreme cases represented by the gray hatched area. This could be an indication that the gas properties of some regions are still evolving toward dynamical, thermal, and star formation equilibrium. As shown in the pressure distributions computed by Kim et al. (2011, 2013) there is always a significant variation about the equilibrium value. On one hand, $\Sigma_{\text {SFR }}$ varies in time about its mean value, which affects $G_{0}$ and therefore $P_{\text {two-phase }}$. On the other, turbulent compressions and expansions move local regions away from $P_{\text {two-phase }}$.

Another possibility is that part of the observed scatter is driven by observational uncertainties. In particular, $\Sigma_{\mathrm{SFR}}$ measurements below the $\sim 10^{-3} M_{\odot} \mathrm{yr}^{-1} \mathrm{kpc}^{-2}$ level can be unreliable due to large uncertainties in the $\mathrm{H} \alpha$ data and a growing contribution to the $24 \mu \mathrm{m}$ emission from old stars (e.g., Leroy et al. 2012; Draine et al. 2014; Herrera-Camus et al. 2015).

\subsection{Dependence on Metallicity}

In the right panels of Figure 5, we have color coded regions according to the characteristic oxygen abundance of its parent galaxy. For the model comparison, we include results from Equations (13) and (14), assuming $P_{\max } / P_{\min }=2, \Sigma_{\text {gas }}=10$ $M_{\odot} \mathrm{pc}^{-2}$ and three different values for the dust abundance relative to the solar neighborhood, $Z_{d}^{\prime}=0.3$ (solid line), 1 (dashed line), and 3 (dotted-dashed line). The model predicts that for a fixed amount of $\Sigma_{\mathrm{SFR}}$, the thermal pressure increases with decreasing dust abundance $Z_{d}^{\prime}{ }^{17}$ In our sample, we find that for regions with similar $\langle U\rangle$ or $\Sigma_{\mathrm{SFR}}$, those with lower

\footnotetext{
17 Naively, one might expect lower dust abundance to decrease $P_{\text {th }}$ because the photoelectric heating rate decreases. However, if $Z_{g}^{\prime} \propto Z_{d}^{\prime}$, the cooling rate by fine-structure $\mathrm{C}$ and $\mathrm{O}$ lines decreases at a compensating rate, as expressed by the numerator of Equation (13). Since in the scenario of lower $Z_{d}^{\prime}$ the X-ray heating rate would remain unchanged, the overall heating/cooling would increase as $Z_{d}^{\prime}$ decreases, raising the equilibrium pressure as expressed by Equation (14).
} 


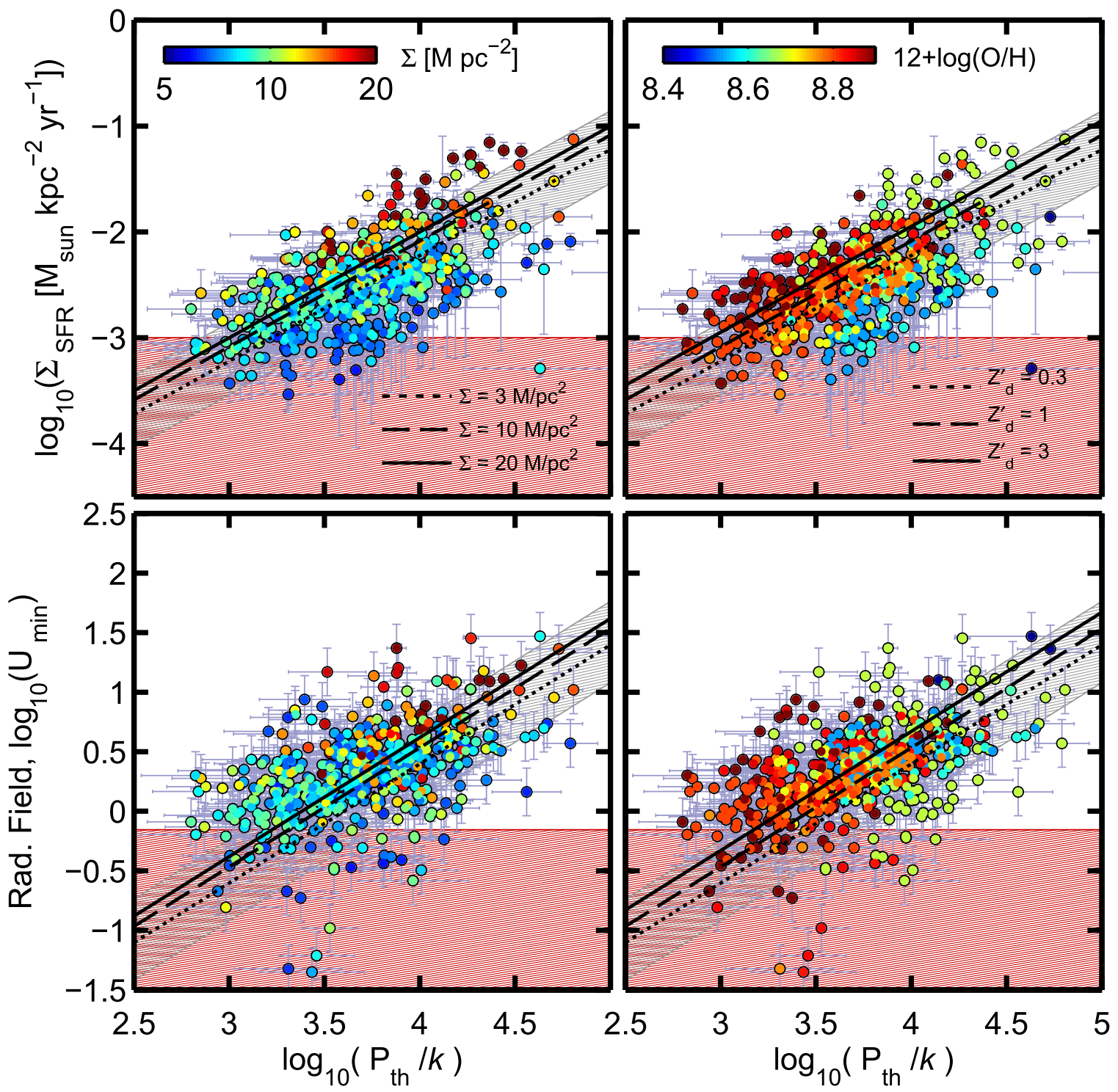

Figure 5. Thermal pressure vs. star formation surface density (upper panels) and radiation field strength (lower panels) for regions with $R_{\text {mol }} \leqslant 1$ in the KINGFISH sample. (Left panels) The color scale represents the gas surface density $\Sigma_{\text {gas }}=\Sigma_{\mathrm{HI}_{\mathrm{I}}}+\Sigma_{\mathrm{H}_{2}}$. The results from the Wolfire et al. (2003) model (Equation (14) and Equation (15)) when assuming $P_{\max } / P_{\min }=2, Z_{d}^{\prime}=1$ and $\Sigma_{\mathrm{gas}}=3,10$ and $20 M_{\odot} \mathrm{pc}^{-2}$ are shown as dashed, solid, and dotted lines, respectively. (Right panels) Similar to the left panels, but this time the color scale represents the oxygen abundance taken from Moustakas et al. (2010). The model predictions from Wolfire et al. (2003; Equations (14) and (15)) when assuming $P_{\max } / P_{\min }=2, \Sigma_{\text {gas }}=10 M_{\odot} \mathrm{pc}^{-2}$ and $Z_{d}^{\prime}=0.3,1,3$ are shown using dashed, solid, and dotted lines, respectively. The hatched gray regions represent the maximal area where $P_{\min } \lesssim P_{\mathrm{th}} \lesssim P_{\max }$ based on the range of values assumed for $P_{\max } / P_{\min }, \Sigma_{\text {gas }}$, and $Z_{d}^{\prime}$ (the upper edge is defined by $P_{\max } / P_{\min }=2, \Sigma_{\text {gas }}=20 M_{\odot} \mathrm{pc}^{-2}$ and $Z_{d}^{\prime}=3$, and the lower edge by $P_{\max } / P_{\min }=5, \Sigma_{\text {gas }}=3 M_{\odot} \mathrm{pc}^{-2}$ and $\left.Z_{d}^{\prime}=0.3\right)$. The red hatched boxes mark the regions where our star formation rate surface density or radiation field intensity measurements can be affected by additional uncertainties (e.g., in the case of $\Sigma_{\mathrm{SFR}}$, contribution from old stars). In all panels, we assume that $f_{\text {ion }}=f_{\mathrm{H}_{2} \text {, diff }}=0.3$.

metallicities tend to have higher thermal pressures, consistent with the trend predicted in Equations (13)-(15). Higher thermal pressures compared to the Galactic plane values have also been reported in regions of cold, low metallicity gas in the Magellanic Clouds (Welty et al. 2016).

\section{Comparison between the Thermal and Total Pressure}

In a multiphase ISM in dynamical equilibrium, the midplane thermal and turbulent pressure forces balance the weight of the ISM, $P_{\text {tot }}$, that arises from stars, diffuse gas, and dark matter content (Lockman \& Gehman 1991; Cox 2005; Ostriker et al. 2010). In this context, the ratio between the thermal and total pressure, $\alpha=P_{\text {tot,DE }} / P_{\text {th }}$, indicates whether the pressure of the diffuse ISM is thermally $(\alpha<2)$ or dynamically $(\alpha>2)$ dominated. Hydrodynamical simulations of multiphase galactic disks run by Kim et al. $(2011,2013)$ find an average value of $\alpha \approx 4$ (i.e., the thermal pressure is typically $\sim 25 \%$ of the dynamical equilibrium total pressure). These simulations also find a very weak dependence of $\alpha$ with star formation activity $\left(\alpha \propto\left(\Sigma_{\mathrm{SFR}}\right)^{0.03}\right.$; Kim et al. 2013).

Thanks to the wealth of ancillary data available, we combine tracers of thermal pressure, stellar mass surface density, and dark matter content to directly measure $\alpha$. The complete calculation of $\alpha$ is developed in the Appendix. Briefly, we use the expression for the dynamical equilibrium total pressure $P_{\text {tot,DE }}$ detailed in Kim et al. (2011). In this formulation, $P_{\text {tot,DE }}$ is a function of $\Sigma_{\text {gas }}$, the gas vertical velocity dispersion $\left(\sigma_{z}\right)$, and the midplane density of the stellar disk $\left(\rho_{*}\right)$ and the dark matter halo $\left(\rho_{\mathrm{DM}}\right)$. Figure 6 shows the resulting $P_{\text {tot,DE }}$ as a 


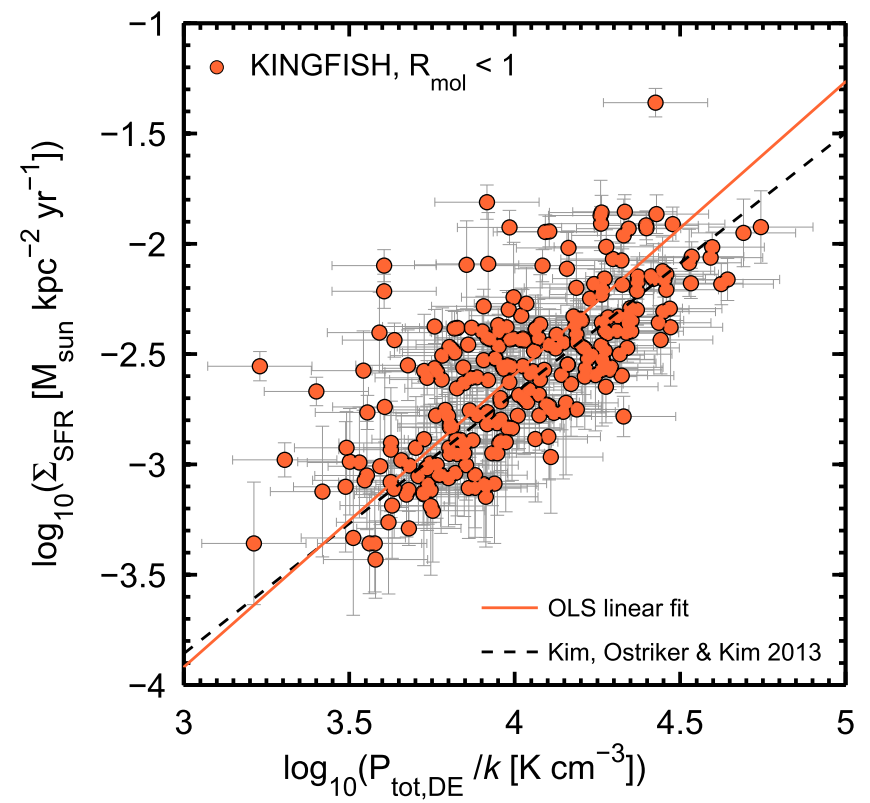

Figure 6. Dynamical equilibrium total pressure $\left(P_{\mathrm{tot}, \mathrm{DE}}\right)$ vs. star formation rate surface density $\left(\Sigma_{\mathrm{SFR}}\right)$ for regions with $R_{\mathrm{mol}} \leqslant 1$ in the KINGFISH sample. The best linear fit to the data, as estimated by the ordinary least-squares (OLS) linear bisector method (Isobe et al. 1990), yields $\log _{10}\left(\Sigma_{\mathrm{SFR}}\right)=$ $1.3 \times \log _{10}\left(P_{\text {tot,DE }} / k\right)-7.9$ (orange line). We also include the scaling relation between $P_{\text {tot,DE }}$ and $\Sigma_{\mathrm{SFR}}$ from the hydrodynamical simulations by Kim et al. (2013; black dotted line).

function of $\Sigma_{\mathrm{SFR}}$. The correlation agrees well with the best fit to the Kim et al. (2013) hydrodynamical simulations (dashed line).

Regarding the ratio between the total and the thermal pressure, Figure 7 shows the median values of $\alpha$ (and the 25th to 75th percentile range) measured in the KINGFISH regions with $R_{\mathrm{mol}} \leqslant 1$ as a function of $\Sigma_{\mathrm{SFR}}$ (assuming $f_{\mathrm{H}_{2} \text {,diff }}=0.3$ ). Consistent with the expectations from the models, we observe no strong dependence between $\alpha$ and $\Sigma_{\mathrm{SFR}}$ in the $10^{-3} \lesssim \Sigma_{\mathrm{SFR}} / M_{\odot} \mathrm{yr}^{-1} \mathrm{kpc}^{-2} \lesssim 10^{-2}$ range, although the dispersion in the data is large. For comparison, we include the approximate $\alpha$ value in the Solar Neighborhood (Jenkins \& Tripp 2001; Wolfire et al. 2003) and the mean $\alpha$ values computed in two-dimensional and three-dimensional hydrodynamical simulations by Kim et al. (2011, 2013), respectively. We have also included results from a new simulation of the solar neighborhood (Kim \& Ostriker 2017) that follows spacetime correlation of supernovae ( $\mathrm{SNe}$ ) with dense and diffuse gas realistically, resolves all thermal phases of the ISM (the hot phase was missing previously), and fully captures the circulation of the galactic fountain. In particular, the amount of radial momentum injection per $\mathrm{SN}\left(p_{*}\right)$ is self-consistently determined by numerically resolving supernova remnant expansion prior to the onset of cooling (Kim \& Ostriker 2015) in contrast to the previous simulations where it was fixed to $p_{*}=3 \times 10^{5} M_{\odot} \mathrm{km} \mathrm{s}^{-1}$.

If we assume $f_{\mathrm{H}_{2} \text {,diff }}=0$, we find a mean $\alpha$ of 2.6 (red dotted line), which is about $\sim 30 \%$ lower than the $\alpha$ values resulting from the simulations. In order to find a better agreement between the $\alpha$ values of our regions, the solar neighborhood and the simulations, we need to assume a gas mass fraction of diffuse ("CO-dark") $\mathrm{H}_{2}$ gas between $f_{\mathrm{H}_{2} \text {, diff }}=0.15$ and 0.3 . In the latter case, we measure a mean $\alpha=3.7$ (red solid line), similar to the mean $\alpha$ found

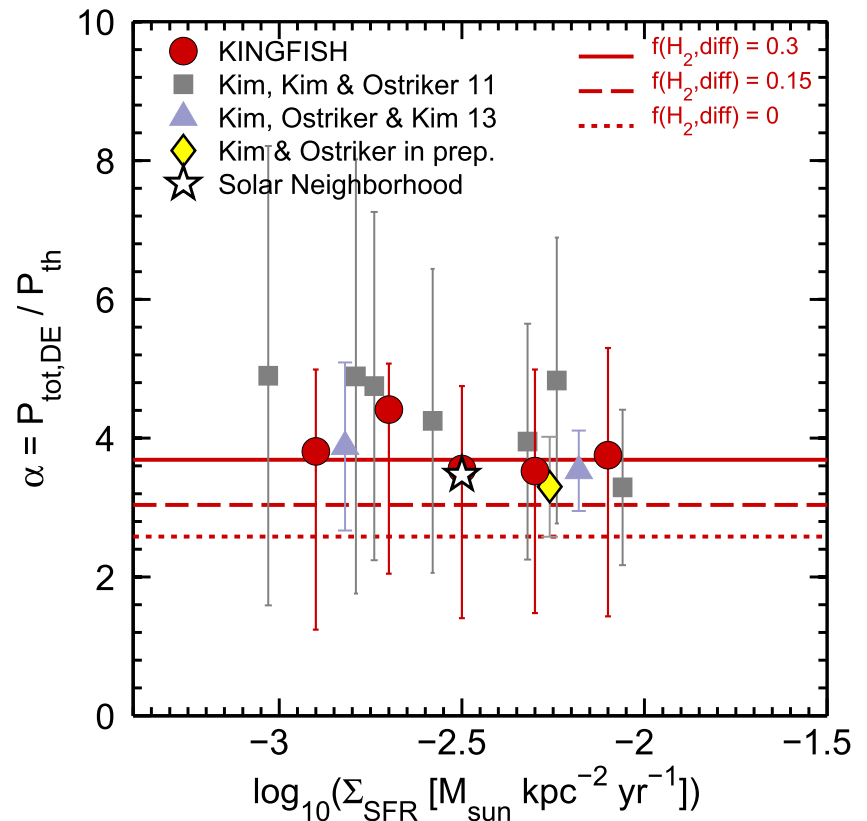

Figure 7. Total-to-thermal pressure ratio $\alpha$ as a function of $\Sigma_{\mathrm{SFR}}$. The red circles show the median $\alpha$ value measured in our KINGFISH sample of regions with $R_{\mathrm{mol}} \leqslant 1$ and assuming $f_{\mathrm{H}_{2} \text {, diff }}=0.3$; the horizontal bars represent the 25 th to 75 th percentile range. The horizontal red line corresponds to the median $\alpha$ value found in our sample across $\Sigma_{\mathrm{SFR}}$. If we change the assumption on $f_{\mathrm{H}_{2} \text {,diff }}$ to 0 (red dotted line) or 0.15 (red dashed line), we find lower median $\alpha$ values. We also include the results from $\mathrm{Kim}$ et al. $(2011,2013)$ hydrodynamical simulations (gray squares and purple triangles, respectively), Kim \& Ostriker (2017; yellow diamond), and the solar neighborhood (open star). The markers represent the mean value and the error bars the $1 \sigma$ standard deviation.

by Kim et al. (2013) and Kim \& Ostriker (2017, in preparation). Independently of our assumption of $f_{\mathrm{H}_{2} \text {, diff }}$, we measure a mean $\alpha$ value in our sample that is $\alpha>2$, implying that the ISM is dominated by dynamical processes.

\section{Summary and Conclusions}

We study the distribution of thermal pressures in the neutral gas of extragalactic regions selected from nearby galaxies that are part of the KINGFISH, HERACLES, and THINGS samples. The method we use to measure the thermal pressure relies on the [C II] $158 \mu \mathrm{m}$ emission that arise from regions where the excitation of $\mathrm{C}^{+}$ions is dominated by collisions with hydrogen atoms (though it is still important to consider the contribution to the total [C II] emission from ionized and diffuse, "CO-dark" $\mathrm{H}_{2}$ gas). In these regions, the $[\mathrm{C} \mathrm{II}]$ and $\mathrm{H} \mathrm{I}$ $21 \mathrm{~cm}$ line emission can be used to measure the cooling rate per hydrogen nucleus $\Lambda_{[\mathrm{C} \text { II] }}$ (Equations (7) and (8)), and then, by assuming a typical temperature for the CNM and a carbon abundance, invert the cooling equation (Equation (9)) to infer CNM volume densities; with these, we then obtain the thermal pressure $P_{\text {th }}$ of the neutral gas (Equation (10)). One advantage of this method is that it is very robust against changes in the assumption of the CNM temperature (Kulkarni \& Heiles 1987).

We highlight the following points.

1. We measure the thermal pressure of the neutral gas in 534 predominantly atomic regions $(\sim 1 \mathrm{kpc}$ in size $)$, where we expect the collisional excitation of $\mathrm{C}^{+}$ions to be dominated by the diffuse, neutral gas. These regions were selected from a larger sample of 2093 extragalactic 
regions with [C II], $\mathrm{H}$ I, and $\mathrm{CO}$ observations available by imposing a cut $R_{\mathrm{mol}}=\Sigma_{\mathrm{mol}} / \Sigma_{\mathrm{H}} \leqslant 1$. The thermal pressure calculations involve a series of assumptions on the properties of the neutral gas and the origin of the [C II] emission, including the fraction that arises from the ionized gas $\left(f_{\text {ion }}\right)$, and the mass fraction of the total column density that corresponds to $\mathrm{H}_{2}$ ("CO-dark") diffuse gas $\left(f_{\mathrm{H}_{2} \text {,diff }}\right)$. We find a thermal pressure distribution that extends from $P_{\mathrm{th}} / k \sim 10^{3}$ to $\sim 10^{5} \mathrm{~K} \mathrm{~cm}^{-3}$.

2. We compare the distribution of thermal pressures in our sample to those measured in the neutral, diffuse gas of Galactic plane. For this, we follow a similar approach to Jenkins \& Tripp (2011) and select a sub-sample of 318 regions with dust-weighted mean starlight intensities $\langle U\rangle \leqslant 3$. We find that the distribution of thermal pressures in this sub-sample can be well represented by a log-normal distribution. The median thermal pressure is $P_{\text {th }} / k \approx 3600 \mathrm{~K} \mathrm{~cm}^{-3}$, a value that is consistent with those from studies of the diffuse ISM in the Galactic plane by Jenkins \& Tripp (2011) and Gerin et al. (2015).

3. The trends observed in the relations between $P_{\text {th }}$ and $\Sigma_{\mathrm{SFR}}$ (and $\langle U\rangle$ ), as a function of $G_{0}, \Sigma_{\text {gas }}$ and metallicity are consistent with the results from Wolfire et al. (1995, 2003) and Ostriker et al. (2010) models. In general, the thermal pressures measured in our regions are consistent with the expectations from a two-phase model in pressure equilibrium, where $P_{\mathrm{th}}$ increases as a function of the radiation field intensity and the star formation activity.

4. We use the thermal pressure together with the midplane gravitational equilibrium pressure of the gas disk to estimate $\alpha=P_{\text {tot,DE }} / P_{\text {th }}$. We measure mean $\alpha$ values of $\alpha=2.6$ and 3.7 after assuming diffuse $\mathrm{H}_{2}$ ("CO-dark") gas mass fractions of $f_{\mathrm{H}_{2} \text {,diff }}=0$ and 0.3 , respectively. Irrespective of the assumption on $f_{\mathrm{H}_{2}, \text { diff }}$, we find that $\alpha>2$, which implies that the ISM in our regions is dynamically rather than thermally dominated. In order to find optimal agreement between the results from our observations and hydrodynamical simulations by Kim et al. $(2011,2013)$ it is necessary to assume that the gas mass fraction of diffuse $\mathrm{H}_{2}$ ("COdark") gas in our regions is $f_{\mathrm{H}_{2} \text {, diff }} \gtrsim 0.15$ and the fraction of the neutral gas in the CNM phase is $f_{\mathrm{CNM}} \approx 0.5$.

We thank the referee for helpful comments and suggestions that improved the paper. We thank C.-G. Kim for useful discussions and comments. R.H.C. acknowledges support from a Fulbright-CONICYT grant. A.D.B. acknowledges partial support from a CAREER grant NSF-AST0955836, from NSFAST1139998, from NASA-JPL 1373858, NSF-AST 1412419 and from a Research Corporation for Science Advancement Cottrell Scholar award. M.G.W. was supported in part by NSF grant AST-1411827. E.C.O. is supported by NSF grant AST1312006. The work of A.K.L. is supported by the National Science Foundation under Grants No. 1615109 and 1615105. PACS has been developed by a consortium of institutes led by MPE (Germany) and including UVIE (Austria); KU Leuven, CSL, IMEC (Belgium); CEA, LAM (France); MPIA (Germany); INAF-IFSI/OAA/OAP/OAT, LENS, SISSA (Italy); IAC (Spain). This development has been supported by the funding agencies BMVIT (Austria), ESA-PRODEX (Belgium), CEA/CNES (France), DLR (Germany), ASI/INAF (Italy), and CICYT/MCYT (Spain). HIPE is a joint development by the
Herschel Science Ground Segment Consortium, consisting of ESA, the NASA Herschel Science Center, and the HIFI, PACS, and SPIRE consortia. This work is based (in part) on observations made with Herschel, a European Space Agency Cornerstone Mission with significant participation by NASA. This research has made use of the NASA/IPAC Extragalactic Database (NED), which is operated by the Jet Propulsion Laboratory, California Institute of Technology, under contract with the National Aeronautics and Space Administration. The National Radio Astronomy Observatory is a facility of the National Science Foundation operated under cooperative agreement by Associated Universities, Inc.

\section{Appendix A \\ Measuring the Total to Thermal Midplane Pressure Ratio $(\alpha)$}

In order to measure $\alpha$, we need to take the ratio between the total $\left(P_{\text {tot }}\right)$ and the thermal pressure $\left(P_{\mathrm{th}}\right)$. The details for the calculation of $P_{\mathrm{th}}$ are described in Section 4. For the total pressure $P_{\text {tot }}$, we use the formulation described in detail in Kim et al. (2011). For an ISM that is dominated by diffuse gas - and where the effective pressure is dominated by the thermal and turbulent terms (i.e., cosmic-ray, magnetic field, and radiation effects are unimportant) - the dynamical equilibrium total pressure, $P_{\text {tot,DE, }}$, can be expressed as (Equation (7) in Kim et al. 2011)

$$
P_{\mathrm{tot}, \mathrm{DE}}=\frac{\pi G \Sigma^{2}}{4}\left\{1+\left[1+\frac{32 \sigma_{z}^{2}}{\pi^{2} G} \frac{\rho_{*, \mathrm{DM}}}{\Sigma^{2}}\right]^{1 / 2}\right\} \text {. }
$$

Here, $\Sigma$ is the total surface density of the gas, $\sigma_{z}$ is the vertical gas velocity dispersion, and $\rho_{* \text {,DM }}$ is the sum of the midplane density of the stellar disk $\left(\rho_{*}\right)$ and the density of the dark matter halo $\left(\rho_{\mathrm{DM}}\right)$. As shown in Kim et al. (2011), Equation (16) can be simplified to

$$
\begin{aligned}
P_{\text {tot,DE }}= & 10^{4} k_{B} \mathrm{~cm}^{-3} \mathrm{~K} \times\left(\frac{\Sigma}{10 M_{\odot} \mathrm{pc}^{-2}}\right) \\
& \times\left[0.33\left(\frac{\Sigma}{10 M_{\odot} \mathrm{pc}^{-2}}\right)\right. \\
& \left.+1.4\left(\frac{\rho_{*, \mathrm{DM}}}{0.1 M_{\odot} \mathrm{pc}^{-3}}\right)^{1 / 2}\left(\frac{\sigma_{z}}{10 \mathrm{~km} \mathrm{~s}^{-1}}\right)\right],
\end{aligned}
$$

which is the equation we use to calculate $P_{\text {tot,DE }}$ in our sample of regions. Below, we describe how we measure the three key parameters- $\rho_{*, \mathrm{DM}}, \sigma_{z}$, and $\Sigma$ - that go into Equation (17).

\section{A.1. Midplane Density of the Stellar Disk $\left(\rho_{*}\right)$}

For the calculation of $\rho_{*}$, we follow the procedure described in detail in Leroy et al. (2008). This calculation assumes that the exponential stellar scale height of a galaxy, $h_{*}$, does not vary with radius.

In terms of $h_{*}$ and the stellar mass surface density $\left(\Sigma_{*}\right)$, the density of the stellar disk $\rho_{*}$ can be expressed as (van der Kruit 1988)

$$
\rho_{*}=\frac{\Sigma_{*}}{4 h_{*}} .
$$

We measure $h_{*}$ by assuming that it is related to the stellar scale length, $l_{*}$, by $l_{*} / h_{*}=7.3 \pm 2.2$ (Kregel et al. 2002), and 
using stellar scale lengths for our galaxies drawn from Table 4 in Leroy et al. (2008). We measure $\Sigma_{*}$ following Leroy et al. (2008)

$$
\Sigma_{*}=\Upsilon_{*}^{K}\left\langle\frac{I_{K}}{I_{3.6}}\right\rangle \cos i I_{3.6},
$$

where $\Upsilon_{*}^{K}$ is the $K$-band mass-to-light ratio and $I_{K} / I_{3.6}$ is the $K$ to-3.6 $\mu \mathrm{m}$ intensity ratio. Given the large overlap between our sample and the sample of nearby galaxies in Leroy et al. (2008), we use the same values for $\Upsilon_{*}^{K}$ and $I_{K} / I_{3.6}$ adopted by them, i.e., $\Upsilon_{*}^{K}=0.5 M_{\odot} / L_{\odot, K}$ and $I_{K} / I_{3.6}=1.8$. We determine $I_{3.6}$ from Spitzer $3.6 \mu \mathrm{m}$ maps from SINGS (Kennicutt et al. 2003). Finally, with estimates of $h_{*}$ and $\Sigma_{*}$, we measure $\rho_{*}$ using Equation (18).

\section{A.2. Dark Matter Volume Density $\left(\rho_{\mathrm{DM}}\right)$}

We calculate $\rho_{\mathrm{DM}}$ by assuming a flat rotation curve for the dark halo (i.e., $V_{c}=$ constant), so $\rho_{\mathrm{DM}}$ at a radius $R$ is given by

$$
\rho_{\mathrm{DM}}=\frac{1}{4 \pi G}\left(\frac{V_{c}}{R}\right)^{2} .
$$

We drew the value of $V_{c}$ for our galaxies from Table 4 in Leroy et al. (2008). These velocities were calculated approximating galaxy rotation curves observed in $21 \mathrm{~cm}$ (THINGS, Walter et al. 2008) following a functional form defined in Boissier et al. (2003). We measure the radial distance of our regions taking into account the inclination of the galaxy (Kennicutt et al. 2011; Hunt et al. 2015).

\section{A.3. Vertical Gas Velocity Dispersion $\left(\sigma_{z}\right)$}

For the vertical gas velocity dispersion, we assume a single value of $\sigma_{z}=11 \mathrm{~km} \mathrm{~s}^{-1}$ based on the typical gas velocity dispersion value found for the outer, $\mathrm{H}$ I-dominated parts of THINGS galaxies with inclinations lower than $\sim 60^{\circ}$ (see Figure 21 in Leroy et al. 2008).

\section{A.4. Total Surface Density of the Gas ( $\Sigma)$}

We measure $\Sigma$ as the sum of the atomic $\left(\Sigma_{\mathrm{H}}\right)$ and molecular $\left(\Sigma_{\text {mol }}\right)$ gas mass surface densities. See Sections 2.2 and 2.3 for details of the calculation.

\section{Appendix B Galaxy Sample}

\section{References}

Badhwar, G. D., \& Stephens, S. A. 1977, ApJ, 212, 494

Blitz, L., \& Rosolowsky, E. 2006, ApJ, 650, 933

Boissier, S., Prantzos, N., Boselli, A., \& Gavazzi, G. 2003, MNRAS, 346, 1215

Boselli, A., Gavazzi, G., Lequeux, J., \& Pierini, D. 2002, A\&A, 385, 454 Calzetti, D., Kennicutt, R. C., Engelbracht, C. W., et al. 2007, ApJ, 666, 870 Cox, D. P. 2005, ARA\&A, 43, 337

Crawford, M. K., Genzel, R., Townes, C. H., \& Watson, D. M. 1985, ApJ, 291,755

Croxall, K. V., Smith, J. D., Brandl, B. R., et al. 2013, ApJ, 777, 96

Dale, D. A., Aniano, G., Engelbracht, C. W., et al. 2012, ApJ, 745, 95

Dale, D. A., Cohen, S. A., Johnson, L. C., et al. 2009, ApJ, 703, 517

Dale, D. A., Gil de Paz, A., Gordon, K. D., et al. 2007, ApJ, 655, 863
Draine, B. T. 1978, ApJS, 36, 595

Draine, B. T. 2011, Physics of the Interstellar and Intergalactic Medium (Princeton, NJ: Princeton Univ. Press)

Draine, B. T., Aniano, G., Krause, O., et al. 2014, ApJ, 780, 172

Draine, B. T., Dale, D. A., Bendo, G., et al. 2007, ApJ, 663, 866

Draine, B. T., \& Li, A. 2007, ApJ, 657, 810

Field, G. B., Goldsmith, D. W., \& Habing, H. J. 1969, ApJL, 155, L149

Fuchs, B., Jahreiß, H., \& Flynn, C. 2009, AJ, 137, 266

Gavazzi, G., Boselli, A., Donati, A., Franzetti, P., \& Scodeggio, M. 2003, A\&A, 400, 451

Gerin, M., Ruaud, M., Goicoechea, J. R., et al. 2015, A\&A, 573, A30

Goldsmith, P. F. 2013, ApJ, 774, 134

Goldsmith, P. F., Langer, W. D., Pineda, J. L., \& Velusamy, T. 2012, ApJS, 203, 13

Grenier, I. A., Casandjian, J.-M., \& Terrier, R. 2005, Sci, 307, 1292

Heiles, C., \& Troland, T. H. 2003, ApJ, 586, 1067

Herrera-Camus, R., Bolatto, A. D., Wolfire, M. G., et al. 2015, ApJ, 800, 1

Hoopes, C. G., Walterbos, R. A. M., \& Bothun, G. D. 2001, ApJ, 559, 878

Hunt, L. K., Draine, B. T., Bianchi, S., et al. 2015, A\&A, 576, A33

Isobe, T., Feigelson, E. D., Akritas, M. G., \& Babu, G. J. 1990, ApJ, 364, 104

Jenkins, E. B., \& Tripp, T. M. 2001, ApJS, 137, 297

Jenkins, E. B., \& Tripp, T. M. 2011, ApJ, 734, 65

Kalberla, P. M. W., \& Kerp, J. 2009, ARA\&A, 47, 27

Kennicutt, R. C., Bendo, G., Engelbracht, C., et al. 2003, BAAS, 35, 1351

Kennicutt, R. C., Calzetti, D., Aniano, G., et al. 2011, PASP, 123, 1347

Kennicutt, R. C., Jr., Hao, C.-N., Calzetti, D., et al. 2009, ApJ, 703, 1672

Kennicutt, R. C., Jr., Lee, J. C., Funes, J. G., et al. 2008, ApJS, 178, 247

Kim, C.-G., Kim, W.-T., \& Ostriker, E. C. 2011, ApJ, 743, 25

Kim, C.-G., \& Ostriker, E. C. 2015, ApJ, 815, 67

Kim, C.-G., \& Ostriker, E. C. 2017, ApJ, submitted (arXiv:1612.03918)

Kim, C.-G., Ostriker, E. C., \& Kim, W.-T. 2013, ApJ, 776, 1

Knapen, J. H., Stedman, S., Bramich, D. M., Folkes, S. L., \& Bradley, T. R. 2004, A\&A, 426, 1135

Kobulnicky, H. A., \& Kewley, L. J. 2004, ApJ, 617, 240

Kregel, M., van der Kruit, P. C., \& de Grijs, R. 2002, MNRAS, 334, 646

Kulkarni, S. R., \& Heiles, C. 1987, in Interstellar Processes, Vol. 134, ed. D. J. Hollenbach \& H. A. Thronson, Jr., 87

Langer, W. D., Velusamy, T., Pineda, J. L., Willacy, K., \& Goldsmith, P. F. 2014, A\&A, 561, A122

Leroy, A. K., Bigiel, F., de Blok, W. J. G., et al. 2012, AJ, 144, 3

Leroy, A. K., Walter, F., Bigiel, F., et al. 2009, AJ, 137, 4670

Leroy, A. K., Walter, F., Brinks, E., et al. 2008, AJ, 136, 2782

Leroy, A. K., Walter, F., Sandstrom, K., et al. 2013, AJ, 146, 19

Lockman, F. J., \& Gehman, C. S. 1991, ApJ, 382, 182

Mathis, J. S., Mezger, P. G., \& Panagia, N. 1983, A\&A, 128, 212

Moustakas, J., Kennicutt, R. C., Jr., Tremonti, C. A., et al. 2010, ApJS, 190,233

Muñoz-Mateos, J. C., Gil de Paz, A., Boissier, S., et al. 2009a, ApJ, 701, 1965

Muñoz-Mateos, J. C., Gil de Paz, A., Zamorano, J., et al. 2009b, ApJ, 703,1569

Nicholls, D. C., Sutherland, R. S., Dopita, M. A., Kewley, L. J., \& Groves, B. A. 2016, arXiv: 1612.03546

Ostriker, E. C., McKee, C. F., \& Leroy, A. K. 2010, ApJ, 721, 975

Pilyugin, L. S., \& Thuan, T. X. 2005, ApJ, 631, 231

Pineda, J. L., Langer, W. D., \& Goldsmith, P. F. 2014, arXiv:1409.0537

Pineda, J. L., Langer, W. D., Velusamy, T., \& Goldsmith, P. F. 2013, A\&A, 554, A103

Sandstrom, K. M., Leroy, A. K., Walter, F., et al. 2013, ApJ, 777, 5

Schlegel, D. J., Finkbeiner, D. P., \& Davis, M. 1998, ApJ, 500, 525

Schruba, A., Leroy, A. K., Walter, F., et al. 2011, AJ, 142, 37

Sheffer, Y., Rogers, M., Federman, S. R., et al. 2008, ApJ, 687, 1075

Simón-Díaz, S., \& Stasińska, G. 2011, A\&A, 526, A48

Skibba, R. A., Engelbracht, C. W., Dale, D., et al. 2011, ApJ, 738, 89

van der Kruit, P. C. 1988, A\&A, 192, 117

Walter, F., Brinks, E., de Blok, W. J. G., et al. 2008, AJ, 136, 2563

Welty, D. E., Lauroesch, J. T., Wong, T., \& York, D. G. 2016, ApJ, 821, 118

Wiesenfeld, L., \& Goldsmith, P. F. 2014, ApJ, 780, 183

Wolfire, M. G., Hollenbach, D., \& McKee, C. F. 2010, ApJ, 716, 1191

Wolfire, M. G., Hollenbach, D., McKee, C. F., Tielens, A. G. G. M., \& Bakes, E. L. O. 1995, ApJ, 443, 152

Wolfire, M. G., McKee, C. F., Hollenbach, D., \& Tielens, A. G. G. M. 2003, ApJ, 587, 278

Zibetti, S., Charlot, S., \& Rix, H.-W. 2009, MNRAS, 400, 1181 